\title{
用于氧还原反应的碳基负载金属单原子催化剂研究进展
}

\author{
郝 策，刘自若，刘 炜，史彦涛 \\ (大连理工大学 化工学院 化学系, 精细化工国家重点实验室, 大连 116024)
}

摘 要: 燃料电池能够将化学能转化为电能, 是一种绿色高效的能量转换装置, 但是受到阴极氧还原反应(ORR)动 力学迟缓的限制, 燃料电池需要使用 Pt 等贵金属作为催化剂, 这就导致其成本显著增加。碳基负载单原子催化剂 (C-SACs) 展现出高原子利用率和高选择性等优异性能。另外, C-SACs 在不同 $\mathrm{pH}$ 环境下都显示出优异的 ORR 催化 活性, 被视为贵金属催化剂的经济替代品。本文介绍了近年来提升 C-SACs 的 ORR 催化性能的策略, 包括选择不 同种类的金属中心原子, 调控金属中心的配位结构以及对载体进行杂原子掺杂。同时介绍了这些 C-SACs 在旋转盘 电极和电池器件中的性能。最后对 C-SACs 在实际应用中的可行性以及潜在的挑战进行了展望和总结。

关 键 词: 单原子催化剂; 氧还原反应; 配位环境; 杂原子掺杂; 综述

中图分类号: 0646 文献标志码: A

\section{Research Progress of Carbon-supported Metal Single Atom Catalysts for Oxygen Reduction Reaction}

\begin{abstract}
HAO Ce, LIU Ziruo, LIU Wei, SHI Yantao
(State Key Laboratory of Fine Chemicals, Department of Chemistry, School of Chemical Engineering, Dalian University of Technology, Dalian 116024, China)

Abstract: Fuel cells are highly efficient and green devices for direct chemical-to-electrical energy conversion. However, limited by slow kinetics of oxygen reduction reaction (ORR) in cathode, fuel cells require catalysts with noble metal like Pt, thus significantly increasing the cost of the fuel cells. Carbon-supported metal single atom catalysts (C-SACs) have excellent properties such as high atom utilization efficiency and selectivity. In addition, C-SACs show high ORR activity under different pH conditions, hence have been recognized as economical candidates to replace noble metal in fuel cells. This article reviewed the strategies used to improve ORR activity of C-SACs, including selecting different kinds of metal single atoms, tailoring coordination structure of metal centers, and heteroatomic doping to substrate. Performances of C-SACs in rotating disk electrode or battery device are also introduced. At last, the feasibility and potential challenges of C-SACs in practical application are prospected and summarized.
\end{abstract}

Key words: single atom catalysts; oxygen reduction reaction; coordination environment; heteroatomic doping; review

化石能源的不可再生性促进了可再生能源的开 发和应用 ${ }^{[1]}$ 。许多先进的电化学储能和转换装置, 如 燃料电池 ${ }^{[2]}$ 和金属-空气电池 ${ }^{[3]}$ 等, 以其能量转换效
率高和污染低等优点, 对生产可持续再生型能源具 有重要意义。氧还原反应(ORR)是上述电化学装置 中的阴极反应，可能通过双电子途径或四电子途径

收稿日期：2020-10-10; 收到修改稿日期：2020-12-07; 网络出版日期：2021-01-07

基金项目: 国家自然科学基金(21677029) National Natural Science Foundation of China(21677029)

作者简介: 郝 策(1974-), 男, 教授. E-mail: haoce@dlut.edu.cn HAO Ce(1974-), male, professor. E-mail: haoce@dlut.edu.cn

通信作者：史彦涛，教授.E-mail: shiyantao@dlut.edu.cn SHI Yantao, professor. E-mail: shiyantao@dlut.edu.cn 
发生反应, 反应式如下 ${ }^{[4]}$ :

双电子途径:

$$
\begin{gathered}
\mathrm{O}_{2}+\mathrm{H}_{2} \mathrm{O}+2 \mathrm{e}^{-} \rightarrow \mathrm{HO}_{2}^{-}+\mathrm{OH}^{-} \\
\mathrm{HO}_{2}^{-}+\mathrm{H}_{2} \mathrm{O}+2 \mathrm{e}^{-} \rightarrow 3 \mathrm{OH}^{-} \text {(碱性) } \\
\mathrm{O}_{2}+2 \mathrm{H}^{+}+2 \mathrm{e}^{-} \rightarrow \mathrm{H}_{2} \mathrm{O}_{2} \\
\mathrm{H}_{2} \mathrm{O}_{2}+2 \mathrm{H}^{+}+2 \mathrm{e}^{-} \rightarrow 2 \mathrm{H}_{2} \mathrm{O} \text { (酸性) }
\end{gathered}
$$

四电子途径:

$$
\begin{gathered}
\mathrm{O}_{2}+2 \mathrm{H}_{2} \mathrm{O}+4 \mathrm{e}^{-} \rightarrow 4 \mathrm{OH}^{-} \text {(碱性) } \\
\mathrm{O}_{2}+4 \mathrm{H}^{+}+4 \mathrm{e}^{-} \rightarrow 2 \mathrm{H}_{2} \mathrm{O} \text { (酸性) }
\end{gathered}
$$

然而, ORR 缓慢的反应动力学却制约着上述电化 学装置的发展 ${ }^{[5-7]}$ 。因此需要开发高活性 ORR 催化剂, 促使反应高效进行。目前商用的燃料电池催化剂含 有大量贵金属, 如 $\mathrm{Pt}, \mathrm{Pd}$ 和 $\mathrm{Ru}$ 等 ${ }^{[8-9]}$, 其价格高昂、 稀缺以及稳定性和抗毒化性较差等缺点限制了贵金属 电催化剂的大规模应用 ${ }^{[10-11]}$ 。因此, 人们需要开发具有 类似贵金属电催化活性和更高耐久性的廉价催化剂。

单原子催化剂(SACs), 以固定在特定载体上的 孤立金属原子作为催化活性位点 ${ }^{[12]}$, 是一种具有发 展潜力的新兴催化剂。2011 年 Qiao 等 ${ }^{[13]}$ 首次报道 了SACs。随后，不同种类的 SACs 被广泛地合成和 研究, 例如 $\mathrm{Pt}^{[14]} 、 \mathrm{Rh}^{[15]} 、 \mathrm{Ir}^{[16]} 、 \mathrm{Pd}^{[17]}$ 等显示出 SACs 作为电催化剂的潜在优势。SACs 在 ORR 催化过程 中也同样适用 ${ }^{[18-20]}$ 。

在 SACs 众多载体中, 碳基以其独特的几何和 电子性质, 如：1)来源广泛, 价格低廉; 2)结构多样 化且可控; 3)化学稳定性优异; 4)良好的电导性和丰 富互通的孔隙结构 ${ }^{[21]}$, 被广泛用作 SACs 的载体。 此外, 杂原子的掺杂(如氮)可以引入针定位点, 固 定金属单原子, 形成金属-氮-碳(M-N-C)结构。杂原 子掺杂的碳还能够对 ORR 电催化过程起到积极作 用 ${ }^{[22-23]}$ 。已有多项研究工作证明 M-N-C SACs 在 ORR 电催化方面的活性。Qiu 等 ${ }^{[24]}$ 制备的 Fe-N-C SACs 的半波电位比商业 $\mathrm{Pt} / \mathrm{C}$ 催化剂高出 $40 \mathrm{mV}$ 。 Zhou 等 ${ }^{[25]}$ 对金属-有机框架材料(MOFs)进行负压 热处理, 得到的 Co SACs 在碱性环境中半波电位达 到 $0.901 \mathrm{~V}\left(v s\right.$. RHE)。Deng 等 ${ }^{[26]}$ 合成的 Fe-N-C SACs 在氢-氧和氢-空气燃料电池中的功率密度分别为 775 和 $463 \mathrm{~mW} \cdot \mathrm{cm}^{-2}$ 。 M-N-C 型 SACs 对于取代贵 金属电催化剂并进行大规模实际应用具有重要意 义。本文综述了用于 ORR 的 C-SACs 的最新研究进 展,主要包括以下几个方面: 1)金属单原子的选择;2) 金属单原子配位结构的调控; 3)杂原子掺杂。最后, 对 C-SACs 在实际应用中的可能性和局限性进行了 总结和展望。

\section{1 金属单原子的选择}

通常认为 SACs 的中心金属原子是 ORR 的实际 活性位点 ${ }^{[27]}$ 。在 ORR 过程中, 中心金属原子的 $\mathrm{d}$ 轨 道与氧气和中间产物的 $\mathrm{p}$ 电子相互作用, 导致氧分 子吸附和电子转移 ${ }^{[12]}$ 。因此, 选择合适的金属原子 中心对于 SACs 催化活性的调控作用最直接有效。 过渡金属 $\mathrm{Fe} 、 \mathrm{Co} 、 \mathrm{Ni}$ 作为 SACs 的金属中心原子被 广泛研究, 对于 Fe SACs 来说, 虽然其 ORR 催化活性 普遍较好, 但存在芬顿反应(Fenton reaction)导致的金 属溶解现象 ${ }^{[28]}$; 而 Co SACs 可以在酸性环境的 ORR 中产生大量的 $\mathrm{H}_{2} \mathrm{O}_{2}{ }^{[29]}$, 很可能参与芬顿反应。 这些现象衍生的稳定性问题促使人们将目光投向其 它种类金属中心原子。对于 SACs 来说, 通过构建适当 的配位环境而将金属活性位点的电子结构调节到合 理的状态, 就有可能使金属中心原子成为特定催化 反应的理想活性位点, 因此原本催化惰性的金属经 过调节配位后也可能存在 ORR 催化活性。

\section{1 铜基 C-SACs}

$\mathrm{Cu}$ 作为 SACs 金属中心催化 ORR 的功能越来越 多地被关注研究。Qu 等 ${ }^{[30]}$ 以沸石咪唑酯骨架材料 (ZIF-8)为前体制备了 Cu-SACs, 在碱性环境 $(0.1 \mathrm{~mol} / \mathrm{L}$ $\mathrm{KOH})$ 中其半波电位为 $0.895 \mathrm{~V}$ (vs. RHE), ORR 活性 优于商用 $\mathrm{Pt} / \mathrm{C}$ 。Jiang 等 ${ }^{[31]}$ 将铜原子固定在硫和氮改 性的碳载体(Cu-SA/SNC)上，通过高角度环形暗场 扫描透射电子显微镜(HAADF-STEM)直接观察到了 原子级分散的 $\mathrm{Cu}$; HAADF-STEM 照片以及 EXAFS 谱中 $\mathrm{Cu}-\mathrm{Cu}$ 信号的缺失证实了 $\mathrm{Cu}-\mathrm{SA} / \mathrm{SNC}$ 中 $\mathrm{Cu}$ 原 子的单分散(图 1(a, b)); 在碱性环境 $(0.1 \mathrm{~mol} / \mathrm{L} \mathrm{KOH})$ 中 Cu-SA/SNC 的半波电位达到 $0.893 \mathrm{~V}$ (vs. RHE), 超 过了商业 Pt/C 催化剂(图 1(d, e)); Cu-SA/SNC 作为锌 空气电池的阴极时, 其最高功率密度 $\left(220 \mathrm{~mW} \cdot \mathrm{cm}^{-2}\right)$ 优于商业 Pt/C 催化剂 $\left(175 \mathrm{~mW} \cdot \mathrm{cm}^{-2}\right.$ )(图 1(f))。根据 X 射线吸收光谱(XAS), 建立了 $\mathrm{Cu}-\mathrm{SA} / \mathrm{SNC}$ 的原子结 构模型, 可以表示为 $\mathrm{Cu}-\mathrm{N}_{4}-\mathrm{C}_{8} \mathrm{~S}_{2}$ (图 1(c))。并通过 X 射线吸收精细结构(XAFS) 分析和密度泛函理论 (DFT)研究认为, 以 $\mathrm{Cu}-\mathrm{N}_{4}-\mathrm{C}_{8} \mathrm{~S}_{2}$ 配位体形式存在的 低价态 $\mathrm{Cu}(+1)$ 是催化 ORR 过程的活性位点。Cui 等 ${ }^{[32]}$ 通过热解酞菁铜的方法合成了 $\mathrm{Cu}$ SACs, 在碱 性环境 $(0.1 \mathrm{~mol} / \mathrm{L} \mathrm{KOH})$ 中对 $\mathrm{ORR}$ 的半波电位为 $0.81 \mathrm{~V}\left(v s\right.$. RHE)。Bai 等 ${ }^{[33]}$ 通过热解制备了负载于石 墨烯的铜单原子催化材料(Cu@NG), 在碱性环境中 起始电位为 $0.94 \mathrm{~V}$, 半波电位为 $0.84 \mathrm{~V}$ (vs. RHE)。Wang 等 ${ }^{[34]}$ 在合成过程中通过加入硫脲来调整单个铜原 子配位数, 获得了原子分散的 Cu- $\mathrm{N}_{x}$ 结构(图 2(a))。 结合 XPS 分析, 加入硫脲的 $\mathrm{Cu}-\mathrm{NGS}$ 中 $\mathrm{Cu}^{+} / \mathrm{Cu}^{2+}$ 的 

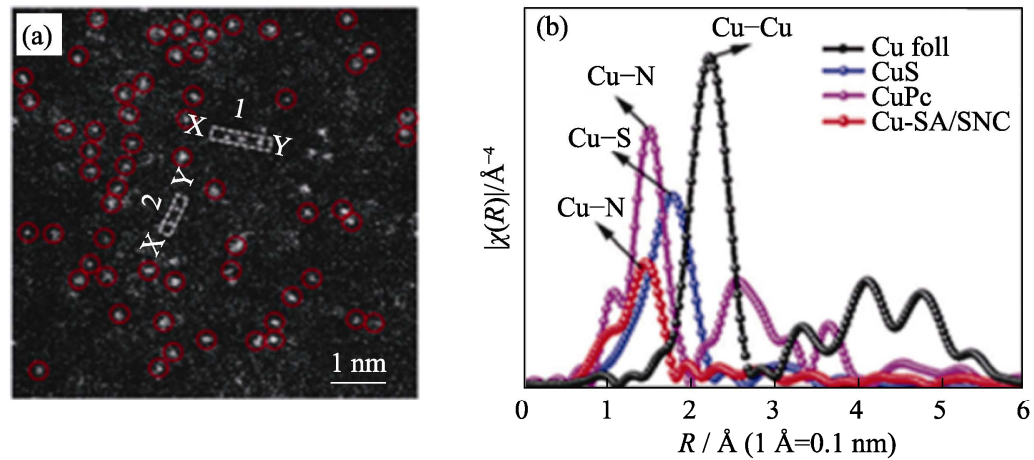

(c)
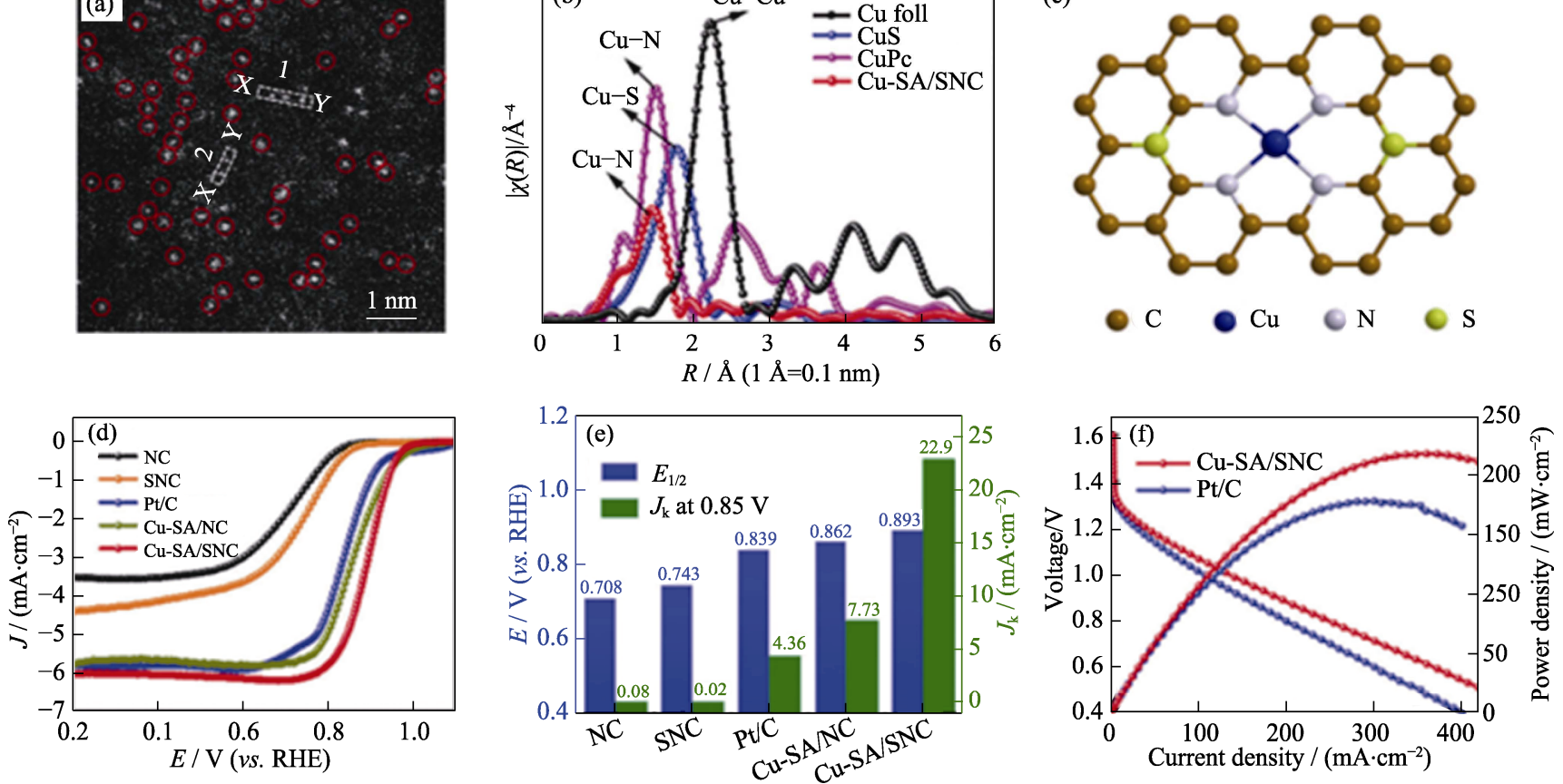

图 1 (a)Cu-SA/SNC 的 HAADF-STEM 照片; (b)Cu-SA/SNC 的 EXAFS 图谱; (c) Cu-SA/SNC 的原子结构示意图; (d)各种催化 剂的 ORR 极化曲线; (e)各催化剂在 $0.85 \mathrm{~V}$ 时动态电流密度和半波电位的比较; (f) 不同锌空气电池的性能测试 ${ }^{[31]}$

Fig. 1 (a) Magnified HAADF-STEM image of Cu-SA/SNC; (b) Ex situ FT $k^{3}$-weighted Cu K-edge EXAFS spectra of Cu-SA/SNC; (c) Schematic interfacial model of Cu-SA/SNC; (d) ORR polarization curves of different catalysts; (e) Kinetic currents at $0.85 \mathrm{~V}$ (vs. RHE) and half-wave potentials of Cu-SA/SNC and their references; (f) Performances of Cu-SA/SNC and Pt/C-based Zn-air batteries ${ }^{[31]}$

Colorful figures are available on website
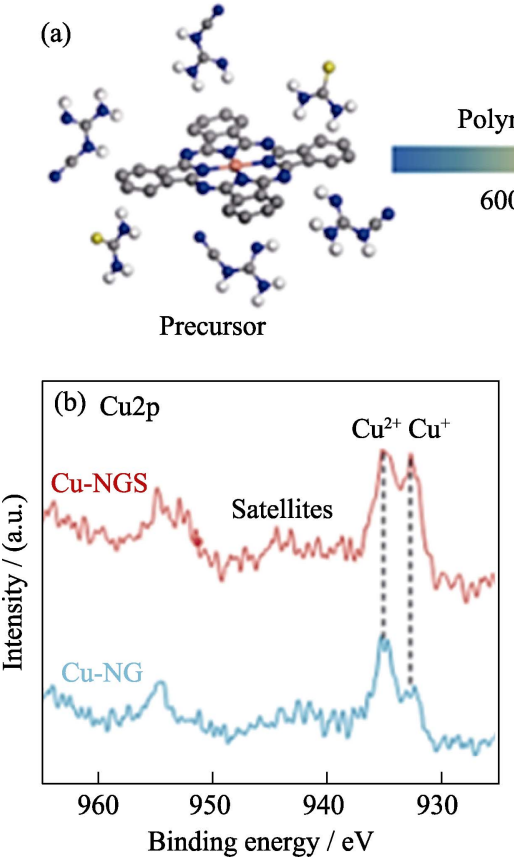

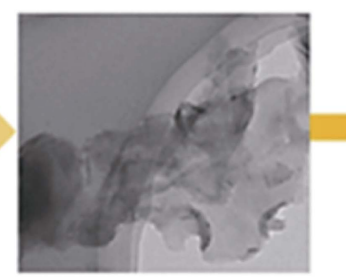

$\mathrm{Cu}$ dope $\mathrm{g}-\mathrm{C}_{3} \mathrm{~N}_{4}$

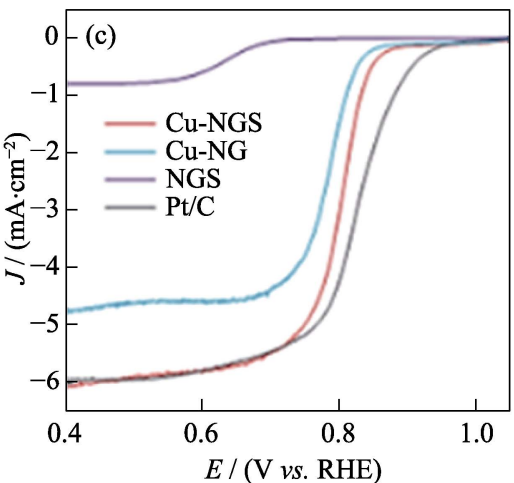

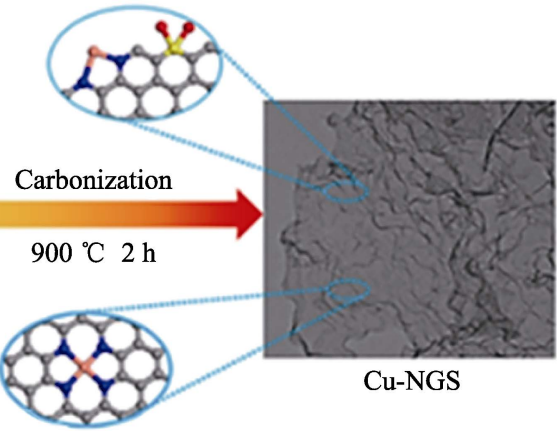

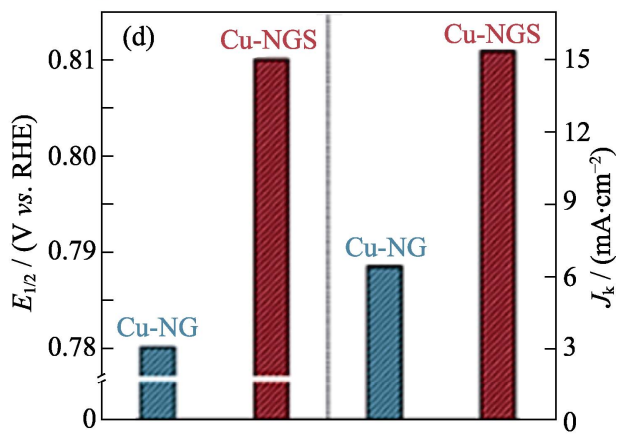

图 $2 \mathrm{Cu}-\mathrm{NGS}$ 的合成示意图、结构表征和电化学测试 ${ }^{[34]}$

Fig. 2 Schematic, characterization and electrochemical test of atomically dispersed $\mathrm{Cu}-\mathrm{N}_{x}$ site catalyst synthesis ${ }^{[34]}$ (a) Schematic of atomically dispersed Cu- $\mathrm{N}_{x}$ site catalyst synthesis; (b) Cu2p XPS spectra; (c) Steady-state ORR polarization plots; (d) Kinetic currents at $0.78 \mathrm{~V}$ (vs. RHE) and half-wave potentials of Cu-NGS and Cu-NG

Colorful figures are available on website

比值增大(图 2(b)), $\mathrm{Cu}-\mathrm{N}_{2} / \mathrm{Cu}-\mathrm{N}_{4}$ 的比例增大, 即配 位不饱和 $\mathrm{Cu}-\mathrm{N}_{2}$ 位点随着硫腿加入量增大而增加。
相关性能测试表明, ORR 活性随 $\mathrm{Cu}-\mathrm{N}_{2}$ 比例的增加 而明显增强(图 2(c, d))。 
为了得到较高负载量的 SACs, Han 等 ${ }^{[35]}$ 在一个铜 容器中对氧化石墨烯和双氧胺进行热解。双氧胺分解成 高活性含氮气体与铜容器反应, 生成含铜的气态前体并 在石墨烯上形成大量分散的 $\mathrm{Cu}$ 单原子, 得到的 $\mathrm{Cu} / \mathrm{G}$ 催 化剂中 $\mathrm{Cu}$ 的负载量达到 5.4wt\%, 并对 ORR 表现出显 著的活性, 在碱性环境 $(0.1 \mathrm{~mol} / \mathrm{L} \mathrm{KOH})$ 中半波电位为 $0.847 \mathrm{~V}$ (vs. RHE)。Wagh 等 ${ }^{[36]}$ 将单原子铜负载在中空氮 化碳纳米球中制备Cu SACs(CuSA@HNCN $\left.{ }_{x}\right)$, 在碱性环 境 $(0.1 \mathrm{~mol} / \mathrm{L} \mathrm{KOH})$ 中 $\mathrm{ORR}$ 半波电位为 $0.91 \mathrm{~V}(v s . \mathrm{RHE})$ 。 此外, 采用 CuSA@ $\mathrm{HNCN}_{x}$ 作为阴极的碱性锌空气 电池具有较高功率密度 $\left(212 \mathrm{~mW} \cdot \mathrm{cm}^{-2}\right)$ 。

Yang 等 ${ }^{[37]}$ 使用气体输送策略合成了孤立的单 原子铜位点 $(\mathrm{Cu}$ ISAS/NC)催化剂, 如图 3 所示, 首 先工业氧化亚铜粉末在接近熔点 $(1500 \mathrm{~K})$ 下升华为 流动蒸汽, 随后被缺陷丰富的含氮碳( $\mathrm{NC}$ ) 捕获并还 原, 形成 Cu SACs(图 3(a))。AC HAADF-STEM 表 征结果显示, 氮掺杂碳上出现了高密度的独立 $\mathrm{Cu}$ 单原子(图 3(b, c))。得到的 Cu ISAS/NC 在碱性环境 $(0.1 \mathrm{~mol} / \mathrm{L} \mathrm{KOH})$ 中半波电位达到 $0.92 \mathrm{~V}(v s$. RHE) (图 3(d)), 远远超过商业 Pt/C 催化剂(半波电位为 $0.846 \mathrm{~V})$ 。采用 $\mathrm{Cu}$ ISAS/NC 作为阴极的锌空气电池, 最高功率密度达到 $280 \mathrm{~mW} \cdot \mathrm{cm}^{-2}$ (图 3(e))。

\section{2 锰基 C-SACs}

芬顿反应会导致稳定性问题，锰与 $\mathrm{H}_{2} \mathrm{O}_{2}$ 之间的
反应活性要弱于 $\mathrm{Fe} / \mathrm{Co}$ 与 $\mathrm{H}_{2} \mathrm{O}_{2}$ 之间的反应活性 ${ }^{[29]}$, 因 此 $\mathrm{Mn}$ 也可以作为 SACs 的金属中心原子。 $\mathrm{Li}^{\text {等 }}{ }^{[38]}$ 将 $\mathrm{Mn}$ 掺杂的 ZIF-8 前体高温碳化, 获得的 $\mathrm{MnN}_{4}$ 单原 子催化材料, 在酸性介质 $\left(0.5 \mathrm{~mol} / \mathrm{L} \mathrm{H}_{2} \mathrm{SO}_{4}\right)$ 中半波电位 达到 $0.8 \mathrm{~V}$ ( vs. RHE), 并且具有良好的稳定性: 经 30000 圈 CV 循环后, 其半波电位的损失只有 $17 \mathrm{mV}$ 。 Yang 等 ${ }^{[39]}$ 对掺 Mn 的 MOFs 进行高温处理, 制备了 $\mathrm{Mn}$ 单原子催化剂, 结构分析发现材料中 $\mathrm{Mn}$ 同时与 $\mathrm{O}$ 原子和 $\mathrm{N}$ 原子形成了 $\mathrm{Mn}-\mathrm{N}_{x} \mathrm{O}_{y}$ 的配位体, 得到的 $\mathrm{Mn} / \mathrm{C}-\mathrm{NO}$ 在碱性条件 $(0.1 \mathrm{~mol} / \mathrm{L} \mathrm{KOH})$ 下的半波电位 达到 $0.86 \mathrm{~V}$ (vs RHE)。Zhu 等 ${ }^{[40]}$ 将氮磷共配位的 $\mathrm{Mn}$ 原子负载到介孔碳材料上, $\mathrm{Mn}$ 以 $\mathrm{MnN}_{x} \mathrm{P}_{y}$ 配位体形式 存在, 得到的 MnNPC-900 在碱性条件 $(0.1 \mathrm{~mol} / \mathrm{L} \mathrm{KOH})$ 下起始电位 $0.97 \mathrm{~V}$, 半波电位 $0.84 \mathrm{~V}$ (vs. RHE)。Bai 等 ${ }^{[41]}$ 将原子分散的锰嵌入到氮掺杂石墨烯中, 得到 的催化剂 Mn@NG 在碱性条件 $(0.1 \mathrm{~mol} / \mathrm{L} \mathrm{KOH})$ 中起 始电位和半波电位分别为 0.97 和 $0.82 \mathrm{~V}$ (vs. RHE)。 Lin 等 ${ }^{[42]}$ 先使 ZIF-8 在水-甲醇混合溶剂中吸附锰离子, 再对其进行一步热解, 得到 Mn-SA。该催化剂具有较 好的半波电势 (0.87 V (vs. RHE)), 在碱性条件 $(0.1 \mathrm{~mol} / \mathrm{L} \mathrm{KOH})$ 下优于商用 $\mathrm{Pt} / \mathrm{C}$ 催化剂。以 $\mathrm{Mn}-\mathrm{SA}$ 为 阴极的锌空气电池在 $0.6 \mathrm{~V}$ 电压下的最大功率密度接 近 $150 \mathrm{~mW} \cdot \mathrm{cm}^{-2}$, 在电流密度为 $20 \mathrm{~mA} \cdot \mathrm{cm}^{-2}$ 下, 经过 $30000 \mathrm{~s}$ 循环后电压无明显变化。
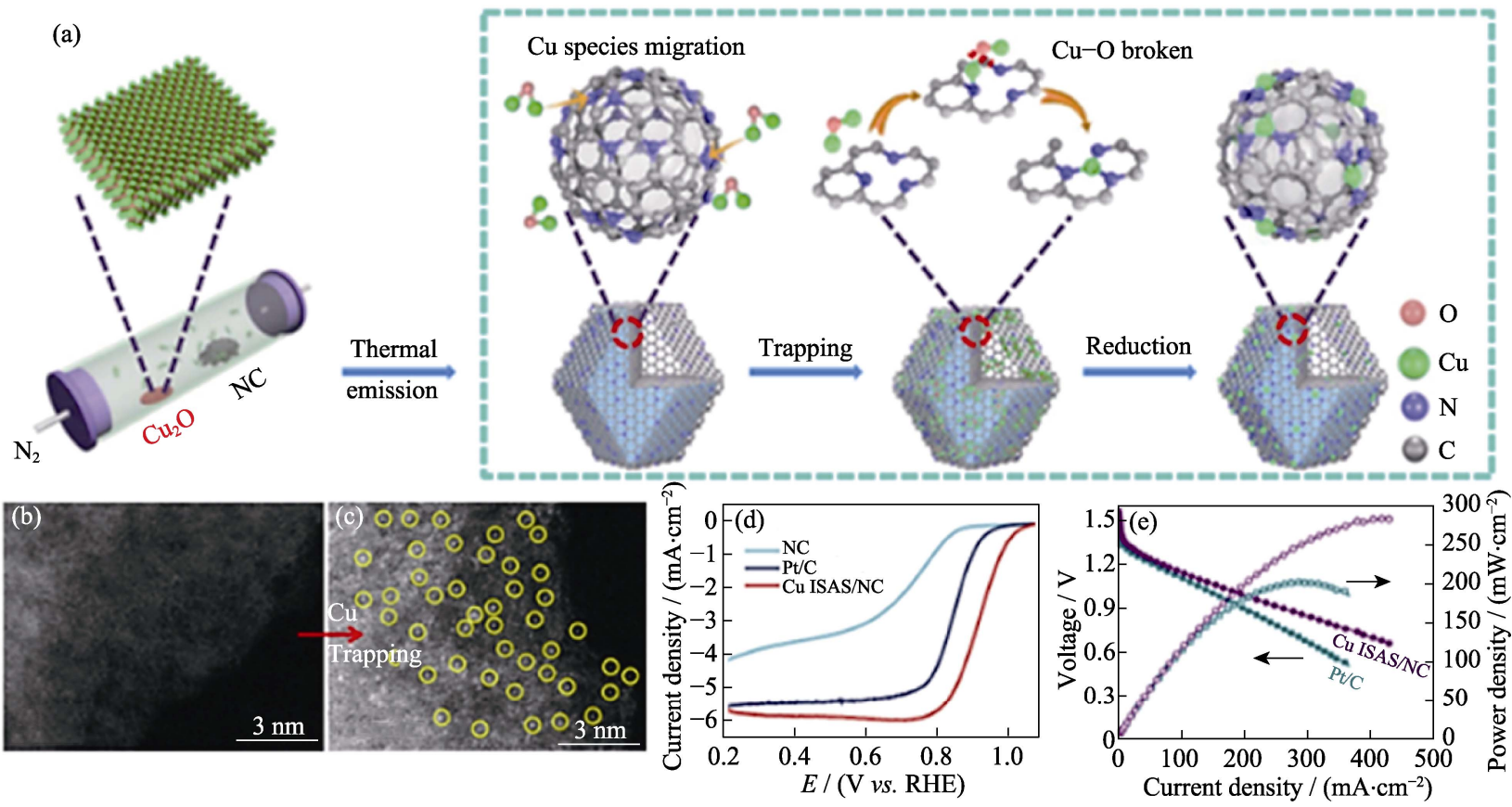

图 3 (a)Cu ISAS/N-C 的形成过程; (b)NC 以及(c)Cu ISAS/NC 的 AC HAADF-STEM 照片;

(d)催化剂的 ORR 极化曲线; (e)不同锌空气电池的性能测试 ${ }^{[37]}$

Fig. 3 (a) Scheme of the formation of Cu ISAS/N-C catalyst; Aberration-corrected high-angle annular dark-field scanning transmission electron microscope (AC HAADF-STEM) images of (b) NC and (c) Cu ISAS/NC;

(d) ORR polarization curves and (e) performances of Cu ISAS/NC and Pt/C-based Zn-air batteries ${ }^{[37]}$ 


\section{3 锌基 C-SACs}

M-N-C 型单原子 ORR 催化剂的过渡金属源主 要限于 $\mathrm{Fe} 、 \mathrm{Co} 、 \mathrm{Ni}$ 等, 目前认为这些过渡金属离子 (例如 $\mathrm{Fe}^{2+} 、 \mathrm{Fe}^{3+}$ 以及过渡金属的中间价)和一些不完 全配位的离子会在 ORR 过程中降低电极和电解质 膜的稳定性。与 $\mathrm{Fe} 、 \mathrm{Co} 、 \mathrm{Ni}$ 等元素相比, $\mathrm{Zn}$ 元素的 $\mathrm{d}$ 轨道完全充满 $\left(3 \mathrm{~d}^{10} 4 \mathrm{~s}^{2}\right)$, 难以形成高价态的氧化离

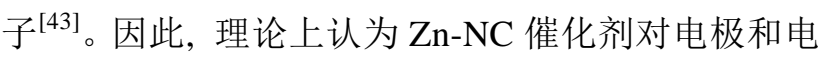
解质膜无害。Song 等 ${ }^{[44]}$ 制备了以 $\mathrm{Zn}-\mathrm{N}_{4}$ 形式分散的 单原子锌催化剂, 由于 $\mathrm{Zn}$ 前驱体在高温热解过程 中挥发性高, $\mathrm{Zn}$ 负载量仅为 $0.3 \mathrm{wt} \%$ 。 $\mathrm{Li}$ 等 ${ }^{[45]}$ 通过控 制 Zn 前驱体的气化速率, 增加了催化剂中单原子 $\mathrm{Zn}$ 的负载, 达到 $9.33 \mathrm{wt} \%(2.06 \mathrm{at} \%)$ 负载。 $\mathrm{Zn}-\mathrm{N}-\mathrm{C}-1$ 在酸性介质 $\left(0.1 \mathrm{~mol} / \mathrm{L} \mathrm{HClO}_{4}\right)$ 中半波电位为 $0.746 \mathrm{~V}$ (vs. RHE), 与 Fe-NC $\left(E_{1 / 2}=0.743 \mathrm{~V}\right)$ 相当; 在碱性介 质中半波电位达到 $0.873 \mathrm{~V}$, 高于商业 $\mathrm{Pt} / \mathrm{C}$ 的 $0.858 \mathrm{~V}$ 。 在稳定性测试中, Zn-N-C-1 催化剂在酸性介质( $0.1 \mathrm{~mol} / \mathrm{L}$ $\mathrm{HClO}_{4}$ )中经过 1000 次 $\mathrm{CV}$ 循环后, 半波电位衰减为 $19.88 \mathrm{mV}$, 比 Fe-N-C-1 的衰减幅度(31.03 mV)小; 在碱性介质中没有明显变化, 而 Fe-N-C-1 催化剂却 有 $18.67 \mathrm{mV}$ 的电位衰减。XPS 分析表明, Zn-N-C-1 催化剂的 $\mathrm{Zn}-\mathrm{N}_{x}$ 活性位点在酸性介质中循环后仅有 略微下降, 证明 Zn SACs 具有良好的稳定性。

\section{4 其它常规过渡金属基 C-SACs}

铇是元素周期表第六族中的一种 $5 \mathrm{~d}$ 过渡金属, 可以形成多种氧化态, 在结构变化过程中其配位数 从 3 到 6 不等。纯铇金属对氧的束缚十分强烈, 脱 除吸附的 $\mathrm{O}$ 和 $\mathrm{OH}$ 在热力学上较为困难, 因此纯铇 金属几乎没有 ORR 催化活性。但在 M-N-C 体系中, W 原子的化学环境和配位情况发生了变化, 铇 SACs 有可能获得良好的 ORR 催化活性。Chen 等 ${ }^{[43]}$ 通过 调控合成温度, 制备了具有 ORR 催化活性的铇 SACs。 $\mathrm{W}-\mathrm{N}$ 配位数为 5 的 W-N-C 材料 $\left(\mathrm{W}-\mathrm{N}_{5}\right)$ 在碱性环境 $(0.1 \mathrm{~mol} / \mathrm{L} \mathrm{KOH})$ 中具有显著的 ORR 催化活性, 其起 始电位为 $1.01 \mathrm{~V}$ (vs. RHE), 半波电位为 $0.88 \mathrm{~V}$, 超 过了商用 $\mathrm{Pt} / \mathrm{C}$ 催化剂(分别为 $1 \mathrm{~V}$ 和 $0.818 \mathrm{~V}$ ); 在酸 性环境 $\left(0.1 \mathrm{~mol} / \mathrm{L} \mathrm{HClO}_{4}\right)$ 中起始电位达到 $0.87 \mathrm{~V}$, 半 波电位达到 $0.77 \mathrm{~V}$; 其质量活性为 $0.63 \mathrm{~A} / \mathrm{mg}(0.9 \mathrm{~V}$ (vs. RHE), 比商用 $\mathrm{Pt} / \mathrm{C}$ 催化剂高约 5.7 倍。而 W- $\mathrm{N}_{3}$ 和 $\mathrm{W}-\mathrm{N}_{4}$ 构型催化剂在酸性和碱性电解质环境中均 表现出较差的 ORR 活性。

为了解决芬顿反应导致的不稳定性, Luo 等 ${ }^{[46]}$ 使用镉作为金属中心原子, 通过热解吸附镉离子的 ZIF 骨架, 制备了以 $\mathrm{Cr}-\mathrm{N}_{4}$ 形式原子级分散的镉 $\mathrm{SACs}(\mathrm{Cr} / \mathrm{N} / \mathrm{C}-950)$ 。该催化剂在酸性环境 $(0.1 \mathrm{~mol} / \mathrm{L}$
$\mathrm{HClO}_{4}$ )中半波电位为 $0.773 \mathrm{~V}$ ( vs. RHE), 2 万次电位 循环后, $\mathrm{Cr} / \mathrm{N} / \mathrm{C}-950$ 半波电位的衰减(15 mV)低于 $\mathrm{Fe} / \mathrm{N} / \mathrm{C}-950$ 催化剂(31 mV), 显示出良好的电化学 稳定性。

一般认为, 主族金属诸如镁和铝等不具有 ORR 催化活性, 但实际上催化位点的内在活性与它们的 电子状态密切相关, 可以通过改变金属位点的配位 数来调节 ${ }^{[47]}$ 。因此, 通过改变配位环境可以使 $\mathrm{Mg}$ 位点具有 ORR 催化活性。Liu 等 ${ }^{[48]}$ 报道了一种具有 ORR 活性的镁 SACs(图 4), 在碱性环境 $(0.1 \mathrm{~mol} / \mathrm{L}$ $\mathrm{KOH})$ 中起始电位和半波电位分别为 1.03 和 $0.91 \mathrm{~V}$ (vs. RHE)。 Mg-N-C 在酸性电解液中 $(0.1 \mathrm{~mol} / \mathrm{L}$ $\left.\mathrm{HClO}_{4}\right)$ 的 ORR 活性接近商业 $\mathrm{Pt} / \mathrm{C}$, 半波电位可达 $0.79 \mathrm{~V}$ 。DFT 模拟表明, $\mathrm{MN}_{1} \mathrm{C}$ 配位体形态不稳定, $\mathrm{MN}_{3} \mathrm{C}$ 的 ORR 活性差, 对 ORR 没有催化活性。因 此, 对 Mg-N-C 的 DFT 和 XANES 的结果表明, Mg-N-C 中最有可能的高活性部分是 $\mathrm{MN}_{2} \mathrm{C}$ 形态配 位体, 主族金属镁的催化活性可以通过两个 $\mathrm{N}$ 原子 的配位来调节, 进而影响其 ORR 催化活性。

\section{5 贵金属基 C-SACs}

金属钉 $(\mathrm{Ru})$ 不仅在氮气还原反应 ${ }^{[49-50]}$ 、特定醇 类的氧化等 ${ }^{[51]}$ 催化反应中表现良好, 在四电子 ORR 过程中也显示出优秀的催化活性。Zhang 等 ${ }^{[19]}$ 在氮 掺杂的石墨烯上制备了 Ru SACs, Ru-N/G 在酸性环 境 $\left(0.1 \mathrm{~mol} / \mathrm{L} \mathrm{HClO}_{4}\right)$ 中表现出良好的四电子 $\mathrm{ORR}$ 活 性, 起始电位和半波电位分别为 0.89 和 $0.75 \mathrm{~V}$ ( v RHE)。Xiao 等 ${ }^{[52]}$ 通过热解 MOF 材料合成的 $\mathrm{Ru}$
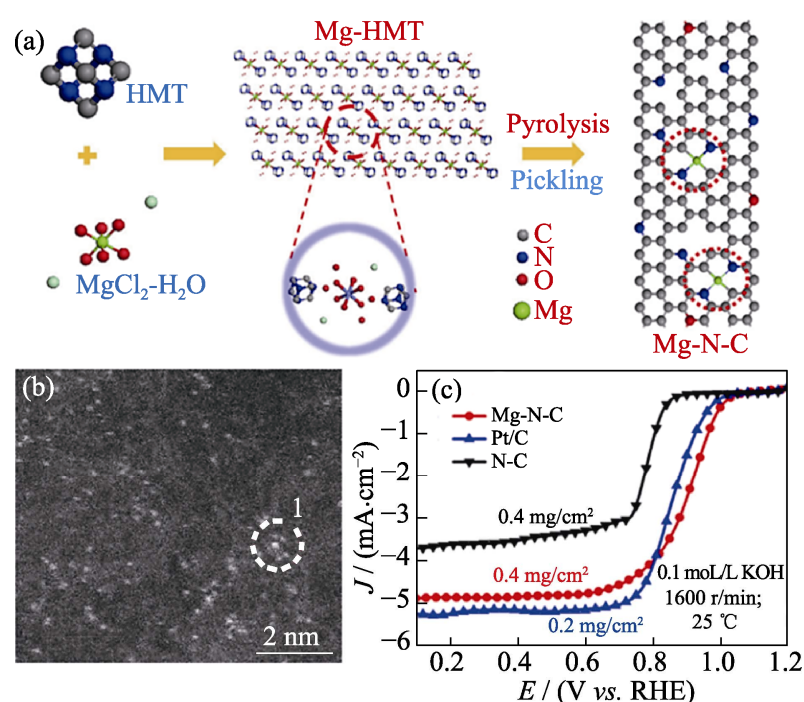

图 4 (a) Mg-N-C 的制备过程示意图, (b) HAADF-STEM 照片 和(c) ORR 极化曲线 ${ }^{[48]}$

Fig. 4 (a) Schematic illustration of the synthesis procedure, (b) HAADF-STEM image and (c) ORR polarization curves of $\mathrm{Mg}-\mathrm{N}-\mathrm{C}^{[48]}$

Colorful figures are available on website 
SACs(Ru-SSC)在酸性环境 $\left(0.1 \mathrm{~mol} / \mathrm{L} \mathrm{HClO}_{4}\right)$ 中, 起 始电位为 $0.92 \mathrm{~V}$ (vs. RHE), 半波电位为 $0.824 \mathrm{~V}$, 与 商业 $\mathrm{Pt} / \mathrm{C}$ 相近。Ru-SSC 的动态电流密度是商业 $\mathrm{Pt} / \mathrm{C}$ 的 1.5 倍, Fe-SSC 的 2.7 倍。另外, 通过紫外-可见 吸收光谱法对 Ru-SSC 的芬顿反应活性进行探针实 验, 发现在 Ru-SSC 上活性氧的生成受到抑制, 因此 该催化剂受芬顿反应干扰较小。Ru-SSC 的半波电位 在 20000 圈电位循环后只有 $18 \mathrm{mV}$ 的负移, 比 Fe-SSC (32 mV) 表现出更优越的稳定性(图 5)。密 度泛函理论(DFT)计算表明, 吸附在 Ru- $\mathrm{N}_{4}$ 位点上 的 $\mathrm{OH}^{*}$ 配体调整了 $\mathrm{Ru}$ 的 $\mathrm{d}$ 轨道电子结构, 优化了 $\mathrm{Ru}$ 位点上含氧中间体的吸附-解吸行为, 从而提高 了催化剂的 ORR 活性。

Xiao 等 ${ }^{[53]}$ 报道了一种 Ir-SAC 用于 ORR 催化, 其质量活性为 $12.2 \mathrm{~A} \cdot \mathrm{mg}_{\text {Ir }}^{-1}$, 显著高于商用 $\mathrm{Pt} / \mathrm{C}$ $\left(0.423 \mathrm{~A} \cdot \mathrm{mg}_{\mathrm{Pt}}^{-1}\right)$ 。 Ir-SAC 在酸性环境 $(0.1 \mathrm{~mol} / \mathrm{L}$ $\mathrm{HClO}_{4}$ ) 中起始电位和半波电位分别为 0.97 和 $0.864 \mathrm{~V}$ (vs. RHE), 优于商业 Pt/C。Liu 等 ${ }^{[54]}$ 将众多贵金属 原子(Ir、Pt、Pd 等)掺杂到沸石咪唑盐骨架(ZIF)中, 再对其热解得到具有 $M-N_{4}$ 活性位点的贵金属 SACs。 其中 Ir SACs 的金属负载量为 $4.5 \mathrm{wt} \%$ 。在酸性环境 $\left(0.1 \mathrm{~mol} / \mathrm{L} \mathrm{HClO}_{4}\right)$ 中 Ir SACs 比纳米颗粒催化剂 $\mathrm{Ir} / \mathrm{C}$ 表现出更高的 ORR 活性, 起始电位和半波电位分 别达到 0.923 和 $0.831 \mathrm{~V}$ (vs. RHE)。通过电化学测试 以及 DFT 计算发现, 合成催化剂的 ORR 活性取决
于配位阴离子的电负性与金属位点表面对 $\mathrm{OH}^{*}$ 的亲 和力是否相配。对于 Ir SACs, $\mathrm{N}_{4}$ 的强电负性可以降 低 $\mathrm{OH}^{*}$ 在 Ir 单原子表面的过强吸附, 因此 Ir SACs 的 ORR 活性比纳米颗粒对应物更高。

作为一种典型的贵金属, 金属 Pt 的 SACs 在 析氢 ${ }^{[55]}$ 等反应中表现优秀。但对于 ORR 反应，金 属 $\mathrm{Pt}$ 粒径减小到单原子尺度后, 过氧化物还原反 应会受到抑制 ${ }^{[56]}$, 对四电子 ORR 过程产生消极作 用, 但对生成 $\mathrm{H}_{2} \mathrm{O}_{2}$ 的双电子 ORR 过程具有很高选 择性 ${ }^{[57-58]}$ 。 Liu 等 ${ }^{[59]}$ 将 $\mathrm{Pt}$ 单原子负载在经 $\mathrm{H}_{2} \mathrm{O}_{2}$ 处 理的缺陷碳上, 得到的 Pt SACs 能够对四电子 ORR 过程起到良好的催化作用, 在酸性环境 $(0.1 \mathrm{~mol} / \mathrm{L}$ $\mathrm{HClO}_{4}$ )中, 半波电位达到 $0.835 \mathrm{~V}$ (vs. RHE), 并具 有较小的 Tafel 斜率 $(93 \mathrm{mV} / \mathrm{dec})$ 。Pt SACs 的 ORR 性能与商用 $\mathrm{Pt} / \mathrm{C}$ 处于同一水平。 Liu 等 ${ }^{[60]}$ 在炭黑 载体上分别制备了 $\mathrm{N}$ 掺杂的 $\mathrm{Pt}_{1}-\mathrm{N} / \mathrm{BP}$ 和无 $\mathrm{N}$ 掺杂 的 $\mathrm{Pt}_{1} / \mathrm{BP}$ 的 Pt SACs(图 6)。EXAFS 研究表明, 氮 掺杂不仅阻止了铂的聚集, 而且抑制了铂原子的 氧化, 避免了 $\mathrm{PtO}_{2}$ 的形成。在酸性条件 $(0.1 \mathrm{~mol} / \mathrm{L}$ $\left.\mathrm{HClO}_{4}\right)$ 下, $\mathrm{Pt}_{1} / \mathrm{BP}$ 的半波电位为 $0.44 \mathrm{~V}$ (vs. RHE), 而掺 $\mathrm{N}$ 的 $\mathrm{Pt}_{1}-\mathrm{N} / \mathrm{BP}$ 的半波电位达到 $0.76 \mathrm{~V}$, 显著的 四电子过程和更低的 $\mathrm{H}_{2} \mathrm{O}_{2}$ 产率意味着 $\mathrm{Pt}_{1}-\mathrm{N} / \mathrm{BP}$ 拥 有良好的 ORR 催化活性。除了碳载体, 在其它载 体上负载的 Pt SACs 对四电子 ORR 也具有较好催 化活性 ${ }^{[61-62]}$ 。
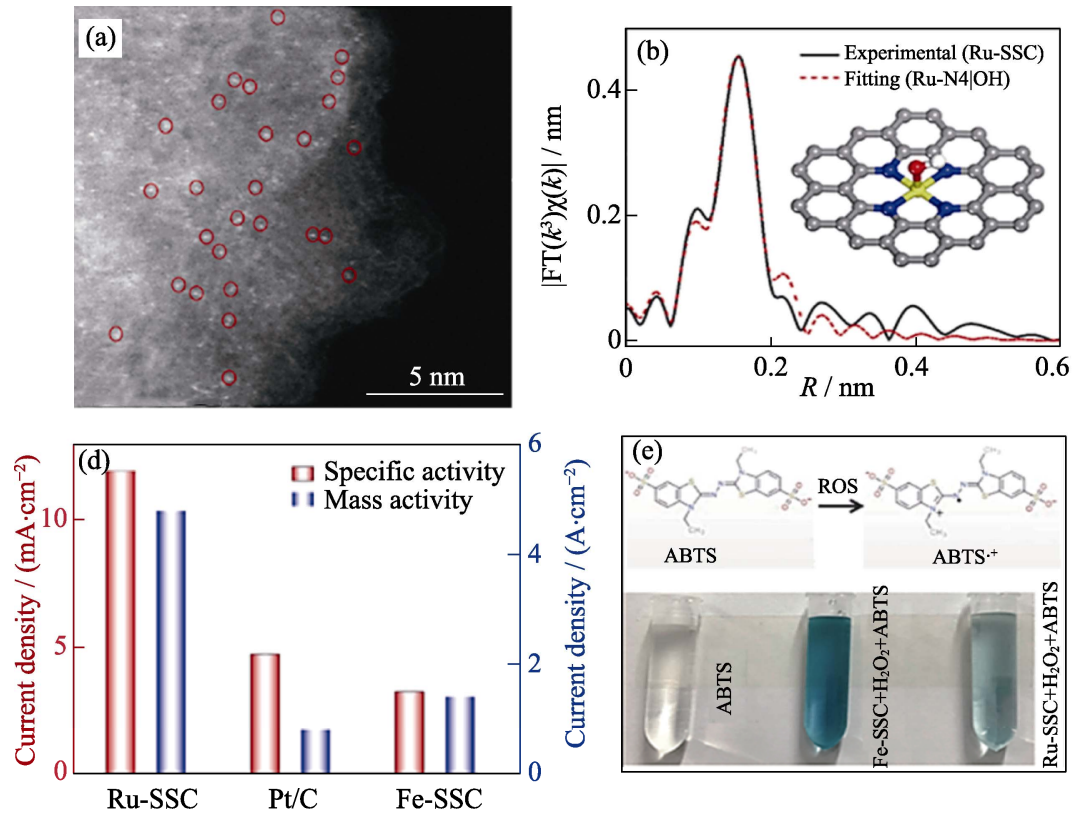
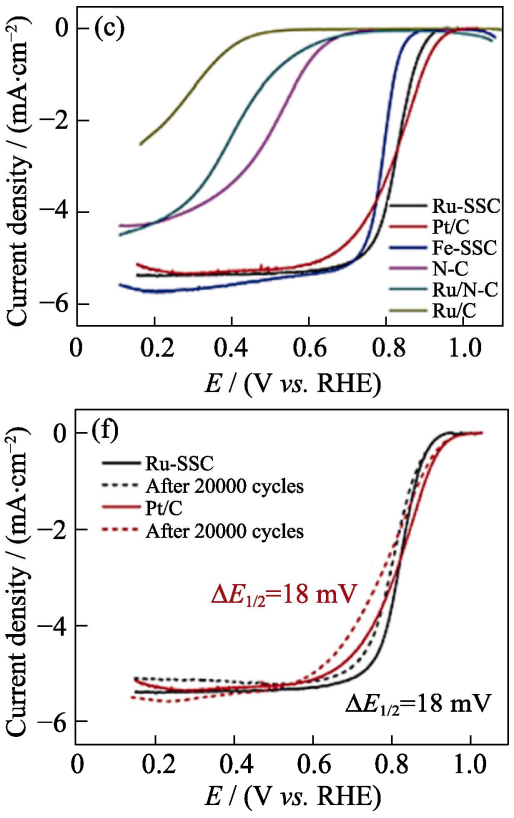

图 $5 \mathrm{Ru}-\mathrm{SSC}$ 的单原子分布、电化学测试以及探针实验 ${ }^{[52]}$

Fig. 5 Atomic dispersion of Ru-SSC, electrocatalytic tests towards ORR and probe experiment ${ }^{[52]}$ (a) High-resolution HAADF-STEM image of Ru-SSC; (b) EXAFS fitting curve for Ru-SSC;(c) ORR polarization curves of the synthesized catalysts; (d) Specific activity and mass activity comparison among Ru-SSC, Pt/C, and Fe-SSC; (e) Reaction between ROS and ABTS (top) and photographs showing the color change of the solution after Fenton reaction (bottom); (f) ORR polarization plots before and after potential cycling stability tests Colorful figures are available on website 

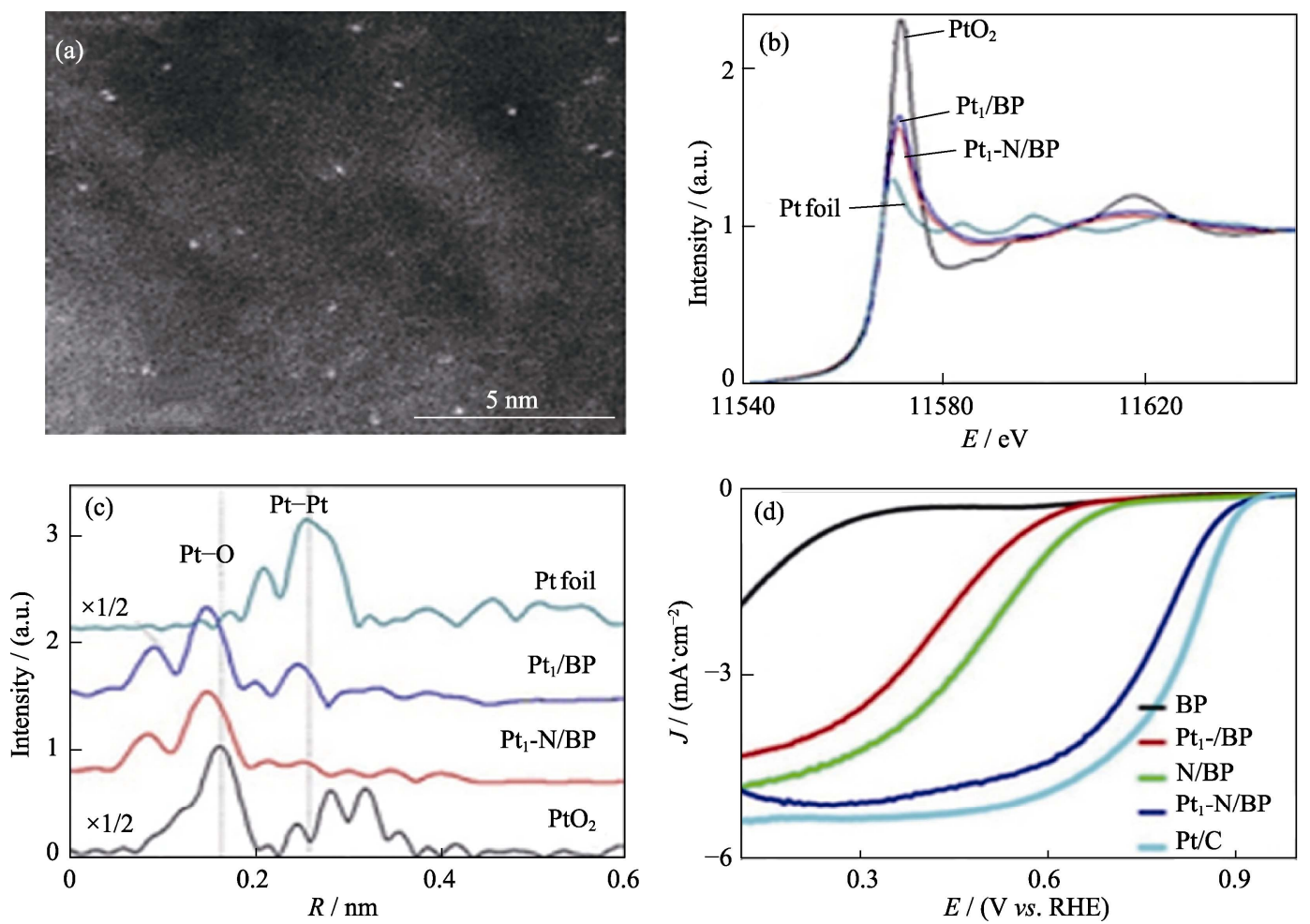

图 6 (a) $\mathrm{Pt}_{1}-\mathrm{N} / \mathrm{BP}$ 的 HAADF-STEM 照片; (b)样品的 XANES, (c) EXAFS 谱和(d) ORR 极化曲线 ${ }^{[60]}$

Fig. 6 (a) HAADF-STEM image of $\mathrm{Pt}_{1}-\mathrm{N} / \mathrm{BP}$, (b) Pt L3-edge XANES, $k^{2}$-weighted $R$-space FT spectra from (c) EXAFS, (d) ORR polarization curves for all samples ${ }^{[60]}$

Colorful figures are available on website

\section{2 金属单原子配位结构的调控}

大量研究表明, 原子级分散的 $\mathrm{M}-\mathrm{N}_{4} / \mathrm{C}$ 催化剂 具有良好的 ORR 催化活性。对配位原子(大多数 $\mathrm{M}-\mathrm{N}-\mathrm{C}$ 结构中为 $\mathrm{N}$ 或 $\mathrm{C}$ )的种类和结构进行调控可 以有效地调节 M-N-C 位点的电子结构 (特别是中心 金属原子的 $\mathrm{d}$ 电子), 使 SACs 获得更好的 ORR 电催 化活性 ${ }^{[63]}$ 。

Han 等 ${ }^{[64]}$ 将原子级分散的二元 Co-Ni 位点嵌入 到氮掺杂的中空碳纳米管 (CoNi-SAs/NC)中, 并确 定其中原子级分散的双金属构型 $\mathrm{N}_{3}-\mathrm{Co}-\mathrm{Ni}-\mathrm{N}_{3}$ 。 $\mathrm{CoNi}-\mathrm{SAs} / \mathrm{NC}$ 在碱性环境 $(0.1 \mathrm{~mol} / \mathrm{L} \mathrm{KOH})$ 中的起始 电位为 $0.88 \mathrm{~V}$, 半波电位为 $0.76 \mathrm{~V}$ (vs. RHE)。

Yuan 等 ${ }^{[65]}$ 将杂原子 $\mathrm{P}$ 引入材料, 制备了氮磷双 配位的铁单原子负载(Fe-N/P-C)碳纳米片。通过 X 射线吸收光谱等技术分析, Fe-N/P-C 中的 $\mathrm{Fe}$ 原子 与 3 个氮原子和 1 个磷原子配位, 在碱性环境 $(0.1 \mathrm{~mol} / \mathrm{L} \mathrm{KOH})$ 中起始电位为 $0.941 \mathrm{~V}$, 半波电位为 $0.867 \mathrm{~V}$ (vs. RHE), 与无磷对照催化剂和商用 $\mathrm{Pt} / \mathrm{C}$ 相比, Fe-N/P-C 具有更好的耐久性和更高的半波电 位(如图 7 所示)。

Zhao 等 ${ }^{[66]}$ 在聚氯乙烯涂覆的碳黑上对铁卟啉 与咪唑进行配位组装, 并热解制备了具有氟杂原子
掺杂的 Fe- $\mathrm{N}_{5}$ SACs, 其在碱性环境 $(0.1 \mathrm{~mol} / \mathrm{L} \mathrm{KOH})$ 中的起始电位为 $0.99 \mathrm{~V}$, 半波电位为 $0.88 \mathrm{~V}(\mathrm{vs}$. RHE), 与未配位咪唑的 C/(DFTPP)Fe-800 和未配位 氟的 C@PVI-(TPP)Fe-800 相比, 表现出更好的 ORR 电催化活性。分析认为, 掺杂引入的氟 C@PVI-(DFTPP)Fe-800 对 ORR 催化活性有积极 影响。

Lin 等 ${ }^{[67]}$ 使用具有螯合配体的金属有机框架来 合成与 5 个 $\mathrm{N}$ 原子配位的金属 SACs。2,2'-双吡啶 可以提供两个非常活跃的 $\mathrm{N}$ 位点结合金属离子, 通 过其与金属前驱物配位和高温处理得到 $\mathrm{Fe}$ 单原子 材料。通过 XANES 光谱分析发现 Fe- $\mathrm{N}_{5}$ 构型的理 论光谱与实验结果吻合。制备的 Fe SAC/N-C 在碱性 环境 $(0.1 \mathrm{~mol} / \mathrm{L} \mathrm{KOH})$ 中的半波电位为 $0.89 \mathrm{~V}$ (vs. RHE), 超过商业 $\mathrm{Pt} / \mathrm{C}$ 催化剂 $40 \mathrm{mV}$, 具有良好的 ORR 催化 活性。

Sun 等 ${ }^{[68]}$ 将硝酸铁和尿素吸附在介孔 $\mathrm{SiO}_{2}$ 包覆 的 ZIF-8 上, 并通过热处理制备了以 $\mathrm{Fe}-\mathrm{N}_{2}$ 形式存在 的单原子 Fe-g- $\mathrm{C}_{3} \mathrm{~N}_{4} / \mathrm{HPNCP}$ 催化剂。结合 EXFAS 表征和 AC-STEM 分析, 证实存在原子分散的 Fe- $\mathrm{N}_{2}$ 配位体。优化后的催化剂 Fe-g- $\mathrm{C}_{3} \mathrm{~N}_{4} / \mathrm{HPNCP}-0.8$ 在 碱性条件 $(0.1 \mathrm{~mol} / \mathrm{L} \mathrm{KOH})$ 下对 $\mathrm{ORR}$ 具有良好的电 催化活性, 半波电位达到 $0.902 \mathrm{~V}$ (vs. RHE), 优于 商业 $\mathrm{Pt} / \mathrm{C}$ 催化剂。 

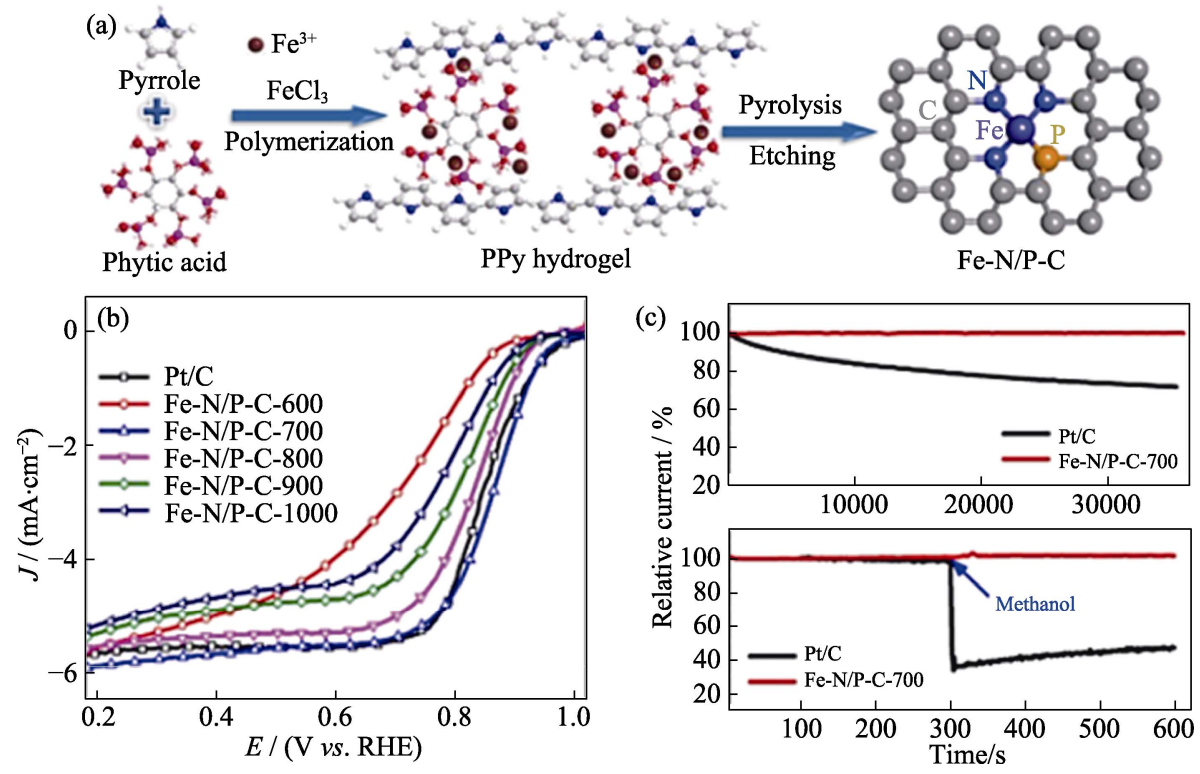

图 7 Fe-N/P-C 的制备过程示意图(a)及其电化学性能表征(b, c) ${ }^{[65]}$

Fig. 7 (a) Schematic diagram of Fe-N/P-C preparation and (b, c) corresponding electrochemical performances ${ }^{[65]}$ Colorful figures are available on website

Zhang 等 ${ }^{[69]}$ 将 $\mathrm{FeCl}_{3}$ 包覆在紫菜表面并进行热 解, 合成了以 $\mathrm{FeN}_{3} \mathrm{~S}_{1}$ 形式原子级分散的 Fe-N-C SACs。该催化剂在碱性环境 $(0.1 \mathrm{~mol} / \mathrm{L} \mathrm{KOH})$ 中半波 电位为 $0.84 \mathrm{~V}$ (vs. RHE), 表现出良好的 ORR 催化 活性。与贵金属催化剂 $\mathrm{Pt} / \mathrm{C}+\mathrm{RuO}_{2}$ 相比, 该电催化 剂组成的可充电锌空气电池具有更高的功率密度 $\left(225.1 \mathrm{~mW} \cdot \mathrm{cm}^{-2}\right)$ 。

Zhang 等 ${ }^{[70]}$ 通过模板辅助的方法合成了一系列 固定在多孔氮、硫共掺杂碳(NSC)基体上的单金属 原子。结构分析表明, 不同金属中心原子的配位环 境有差异: Fe-SAs/NSC 主要由 $\mathrm{FeN}_{4} \mathrm{~S}_{2}$ 配位体组成, 其 中 $\mathrm{S}$ 原子与 $\mathrm{N}$ 原子成键; Co-SAs/NSC 和 Ni-SAs/NSC 中金属中心原子与 $\mathrm{S}$ 原子形成金属 $\mathrm{S}$ 键, 金属单原 子主要以 $\mathrm{CoN}_{3} \mathrm{~S}_{1}$ 和 $\mathrm{NiN}_{3} \mathrm{~S}_{1}$ 配位体形式分散(图 8)。 实验结果表明, Fe-SAs/NSC 的 ORR 电化学性能最 高, 在碱性环境 $(0.1 \mathrm{~mol} / \mathrm{L} \mathrm{KOH})$ 中半波电位达到 $1.00 \mathrm{~V}$ (vs. RHE), 其催化活性优于商业 Pt/C。

Zhao 等 ${ }^{[71]}$ 制备了 Co 单原子负载的 N/P 掺杂介 孔碳纳米球, 其中六氯环三磷腈提供 $\mathrm{N}$ 和 $\mathrm{P}$ 原子, 单宁酸用于配位金属离子。原子结构表征证实了单 原子过渡金属位点 Co 以 Co- $\mathrm{N}_{2} \mathrm{P}_{2}$ 配位体的形式均匀 分布。得到的 PSTA-Co-1000 在碱性环境 $(0.1 \mathrm{~mol} / \mathrm{L}$ $\mathrm{KOH})$ 中半波电位为 $0.878 \mathrm{~V}$ (vs. RHE), 优于同条件 下的商业 $\mathrm{Pt} / \mathrm{C}$ 催化剂。

除了 Fe SACs, 还可改变配位原子来提高 ORR 催化活性。Chen 等 ${ }^{[72]}$ 通过有机聚合物辅助热解法制 备原子分散的 $\mathrm{Cu}$ 催化剂, XPS 和 XAS 谱图证明结 构中的 $\mathrm{N}$ 原子被 $\mathrm{S}$ 原子取代, 形成 $\mathrm{Cu}-\mathrm{N}_{3} \mathrm{~S}_{1}$ 配位体,
得到的 Cu-SA/NPSC 在碱性环境 $(0.1 \mathrm{~mol} / \mathrm{L} \mathrm{KOH})$ 中 的半波电位为 $0.84 \mathrm{~V}$ (vs. RHE)。基于该催化剂研制 的可充电柔性固体锌空气电池具有稳定的开路电压, 最高可达 $1.41 \mathrm{~V}$ 。计算得到 Cu-SA/NPSC 放电过程 中的最大功率密度为 $138.2 \mathrm{~mW} \cdot \mathrm{cm}^{-2}$, 高于 $\mathrm{Pt} / \mathrm{C}$ 的 $121.4 \mathrm{~mW} \cdot \mathrm{cm}^{-2}$, 说明其具有良好的 ORR 性能。

$\mathrm{Ma}$ 等 $^{[73]}$ 通过离子交换法制备了金属中心结构

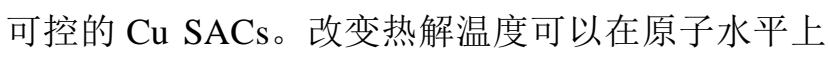
控制活性金属中心的结构, 较高的处理温度 $\left(900{ }^{\circ} \mathrm{C}\right)$ 促进生成不饱和 $\mathrm{Cu}-\mathrm{N}_{3}$ 结构。Cu SAs/ NC-900 在碱 性环境 $(0.1 \mathrm{~mol} / \mathrm{L} \mathrm{KOH})$ 中的半波电位为 $0.87 \mathrm{~V}$, 比

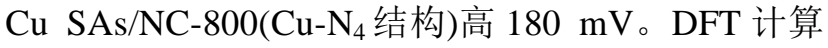
分析进一步表明, $\mathrm{Cu}$ SACs 的低 $\mathrm{N}$ 配位有利于 $\mathrm{O}_{2}{ }^{*}$ 中间体的形成，从而促进 ORR 过程进行。

Sun 等 ${ }^{[74]}$ 通过改变吸附前驱体中的尿素含量来 控制 Cu-SA/NC 催化剂中 $\mathrm{Cu}^{+}$与 $\mathrm{Cu}^{2+}$ 两种氧化态的 比值。热解尿素得到的氨可以作为还原剂使 $\mathrm{Cu}$ 从 +2 价氧化态转化为 +1 价氧化态, 从而引入 $\mathrm{Cu}^{+}-\mathrm{N}$ 位 点。随着 $\mathrm{Cu}^{+}$位点的加入, $\mathrm{Cu}-\mathrm{SA} / \mathrm{NC}$ 催化剂的 ORR 活性增加, 制得的 $\mathrm{Cu}^{+}-\mathrm{SA} / \mathrm{NC}$ (meso)-7(7 指与 $7 \mathrm{~g}$ 尿 素混合)在碱性介质中半波电位为 $0.898 \mathrm{~V}$ (vs. RHE), 显著超过商用 $\mathrm{Pt} / \mathrm{C}$ (如图 9 所示)。

Shang 等 ${ }^{[75]}$ 使用 $\mathrm{SiO}_{2}$ 作为模板, 采用热解法制 备原子构型为 $\mathrm{Mn}-\mathrm{N}_{2} \mathrm{C}_{2}$ 的 SACs (图 10)。EXAFS 拟 合表明, 孤立的 $\mathrm{Mn}$ 原子与两个 $\mathrm{C}$ 原子和两个 $\mathrm{N}$ 原 子相互配位。该 Mn-SAC 在碱性介质中表现出优异 的活性, 半波电位达到 $0.915 \mathrm{~V}(v s$. RHE), 并在 $10 \mathrm{~mA} \cdot \mathrm{cm}^{-2}$ 下具有 $350 \mathrm{mV}$ 的低过电位。 

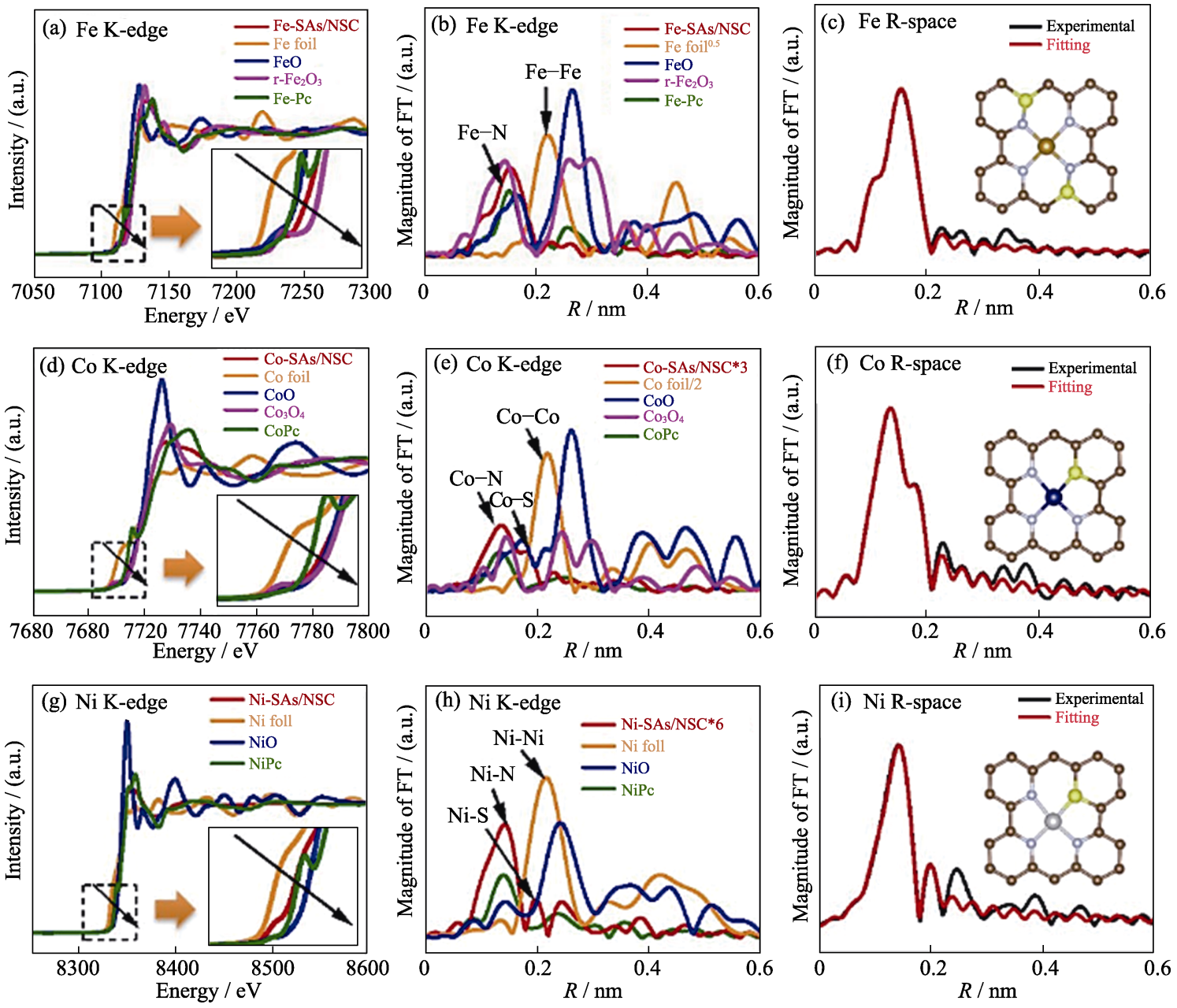

(j)

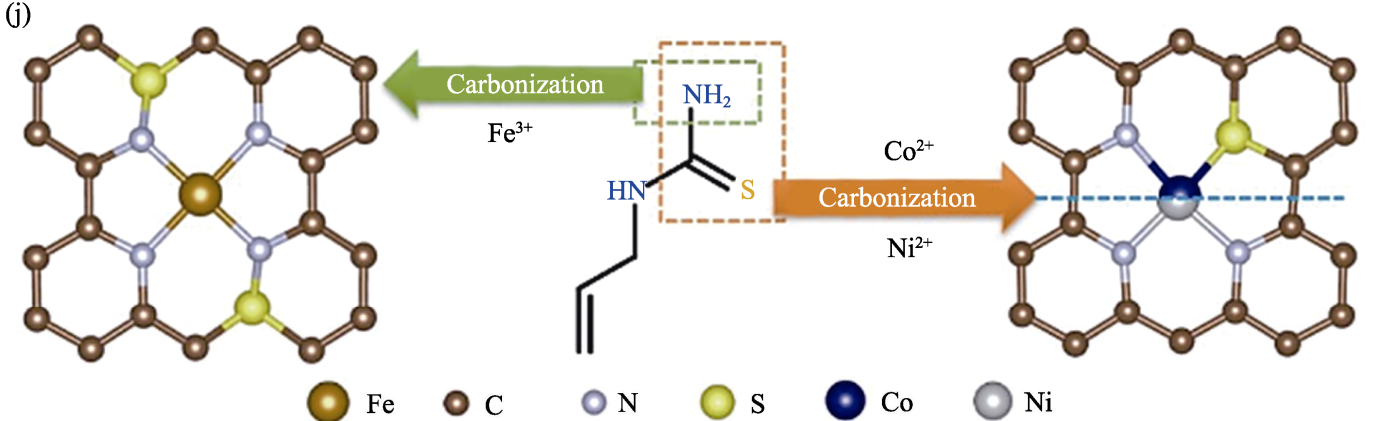

图 8 Fe-SAs/NSC, Co-SAs/NSC 以及 Ni-SAs/NSC 的结构表征 ${ }^{[70]}$

Fig. 8 Structural characterizations of Fe-SAs/NSC, Co-SAs/NSC and Ni-SAs/NSC ${ }^{[70]}$

The normalized XANES spectra and the $k^{3}$-weighted Fourier transform of EXAFS spectra at (a,b) Fe K-edge of Fe-SAs/NSC

and the reference materials, (d,e) Co K-edge of Co-SAs/NSC and the reference materials, and (g,h) Ni K-edge of Ni-SAs/NSC and the reference materials with insets showing the enlarged boxed area in the same picture; EXAFS curves between the experimental data and the fit of (c) Fe-SAs/NSC, (f) Co-SAs/NSC, and (i) Ni-SAs/NSC with insets showing the fitted structures; (j) Schematic illustration of the formation of Fe-SAs/NSC, Co-SAs/NSC, and Ni-SAs/NSC with different coordination environments

Colorful figures are available on website

\section{3 杂原子掺杂}

对于不直接与中心金属原子结合的基底原子, 可以在碳基载体中引入不同的杂原子取代碳原子。 虽然杂原子不直接与金属中心位点结合, 但会通过 远程作用影响 M-N-C 位点的电子结构, 进而改变 ORR 活性。
Sun 等 ${ }^{[76]}$ 提出嗍掺杂可以调节 SACs 的 $\mathrm{d}$ 带中 心, 这有利于活性位点与反应中间物的吸附动力学, 从而提高 ORR 性能。在碱性环境 $(0.1 \mathrm{~mol} / \mathrm{L} \mathrm{KOH})$ 中, 掺杂 B 的催化剂性能优于 Fe-N-C 和商用 $\mathrm{Pt} / \mathrm{C}$, 半波电位达到 $0.933 \mathrm{~V}(v s$. RHE)。通过计算发现 B 掺杂后, 金属中心 $\mathrm{Fe}$ 的价电子增加, 磁矩减小, 这 就造成了良好的氧吸附和较低的过电位, 从而有 

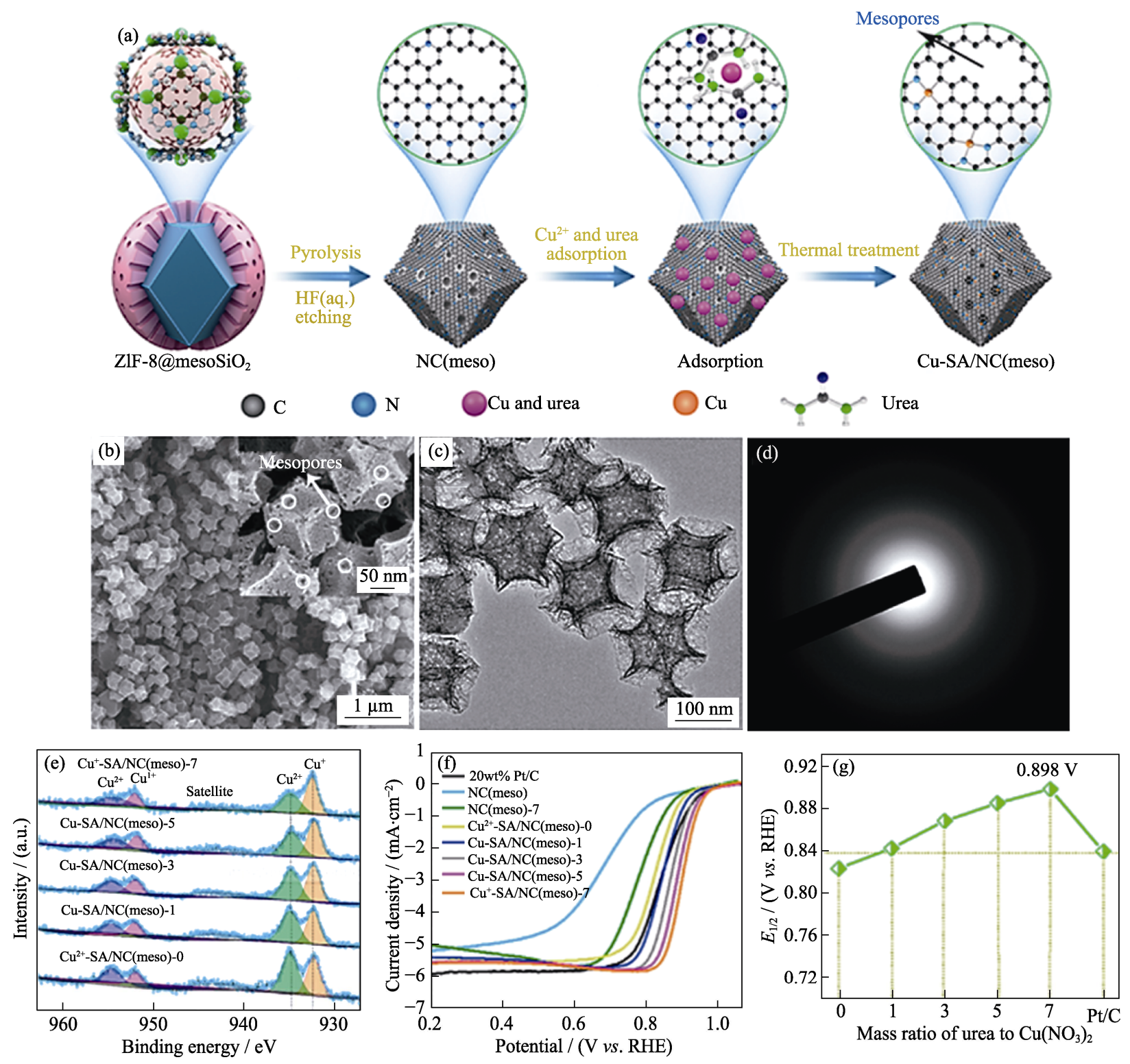

图 9 (a)Cu-SA/NC(meso)制备过程的示意图; $\mathrm{Cu}^{+}-\mathrm{SA} / \mathrm{NC}(\mathrm{meso})-7$ 的(b)SEM 照片, (c)TEM 照片和

(d)选区电子衍射图像; (e)Cu-SA/NC(meso)的高分辨率 XPS 图谱; (f)Cu ${ }^{+}-\mathrm{SA} / \mathrm{NC}(\mathrm{meso})-7$ 以及对比样品

在 $0.1 \mathrm{~mol} / \mathrm{L} \mathrm{KOH}$ 中的 LSV 曲线; (g)Cu-SA/NC(meso)催化剂 ORR 活性的比较 ${ }^{[74]}$

Fig. 9 (a) Formation process of Cu-SA/NC(meso) catalysts; (b) SEM image, (c) TEM image, and (d) corresponding SAED pattern of $\mathrm{Cu}^{+}-\mathrm{SA} / \mathrm{NC}($ meso)-7; (e) Cu2p high-resolution XPS surveys of Cu-SA/NC (meso) catalysts; (f) Linear sweep voltammetry (LSV) curves of $\mathrm{Cu}^{+}-\mathrm{SA} / \mathrm{NC}$ (meso)-7 and comparison samples; (g) Comparison of ORR activities for Cu-SA/NC (meso) catalysts ${ }^{[74]}$

Colorful figures are available on website

利于整个 ORR 过程。除了 B 杂原子掺杂, Sun 等[77] 还通过引入 $\mathrm{P}-\mathrm{O}$ 键来改变单原子位点的催化活性。 密度泛函理论(DFT)模拟验证了 P-O 掺杂改变了 Fe 活性位点的电荷密度分布和电子结构, 并提高了其 对 ORR 和 OER 的催化活性。制备的 P-O/FeN $4-\mathrm{CNS}$ 在碱性环境 $(0.1 \mathrm{~mol} / \mathrm{L} \mathrm{KOH})$ 中半波电位达到 $0.89 \mathrm{~V}$ (vs. RHE), 不仅优于仅用 $\mathrm{P}$ 掺杂的 $\mathrm{P} / \mathrm{FeN}_{4}-\mathrm{CNS}$ $(0.87 \mathrm{~V}(v s . \mathrm{RHE}))$ 和无杂原子掺杂的 $\mathrm{FeN}_{4}-\mathrm{CNS}(0.85 \mathrm{~V}$ (vs. RHE)), 更超过了商业 Pt/C 催化剂(0.85 V(vs. RHE)), 表现出良好的 ORR 催化活性。
Chen 等 ${ }^{[78]}$ 制备了负载在 $\mathrm{N} 、 \mathrm{P}$ 和 S 共掺杂中空 碳多面体上的铁 SACs(Fe-SAs/ NPS-HC)。通过与 N 的近距离配位以及与 $\mathrm{S}$ 和 $\mathrm{P}$ 的长程相互作用, 活性 中心的电子结构得到了调整。制备的 Fe-SAs/ NPS-HC 在碱性环境 $(0.1 \mathrm{~mol} / \mathrm{L} \mathrm{KOH})$ 中半波电位达到 $0.912 \mathrm{~V}$ (vs. RHE); 在酸性环境 $\left(0.5 \mathrm{~mol} / \mathrm{L} \mathrm{H}_{2} \mathrm{SO}_{4}\right)$ 中半波电 位为 $0.791 \mathrm{~V}$ (vs. RHE), 接近商业 Pt/C 催化剂。DFT 计算结果显示, 环境中的 S、P 原子能够向单原子 $\mathrm{Fe}$ 中心提供电子, 使 $\mathrm{Fe}\left(\mathrm{Fe}^{\delta+}\right)$ 的正电荷降低, 从而 削弱与吸附的 $\mathrm{OH}$ 的结合, 进而有助于 ORR 过程。 

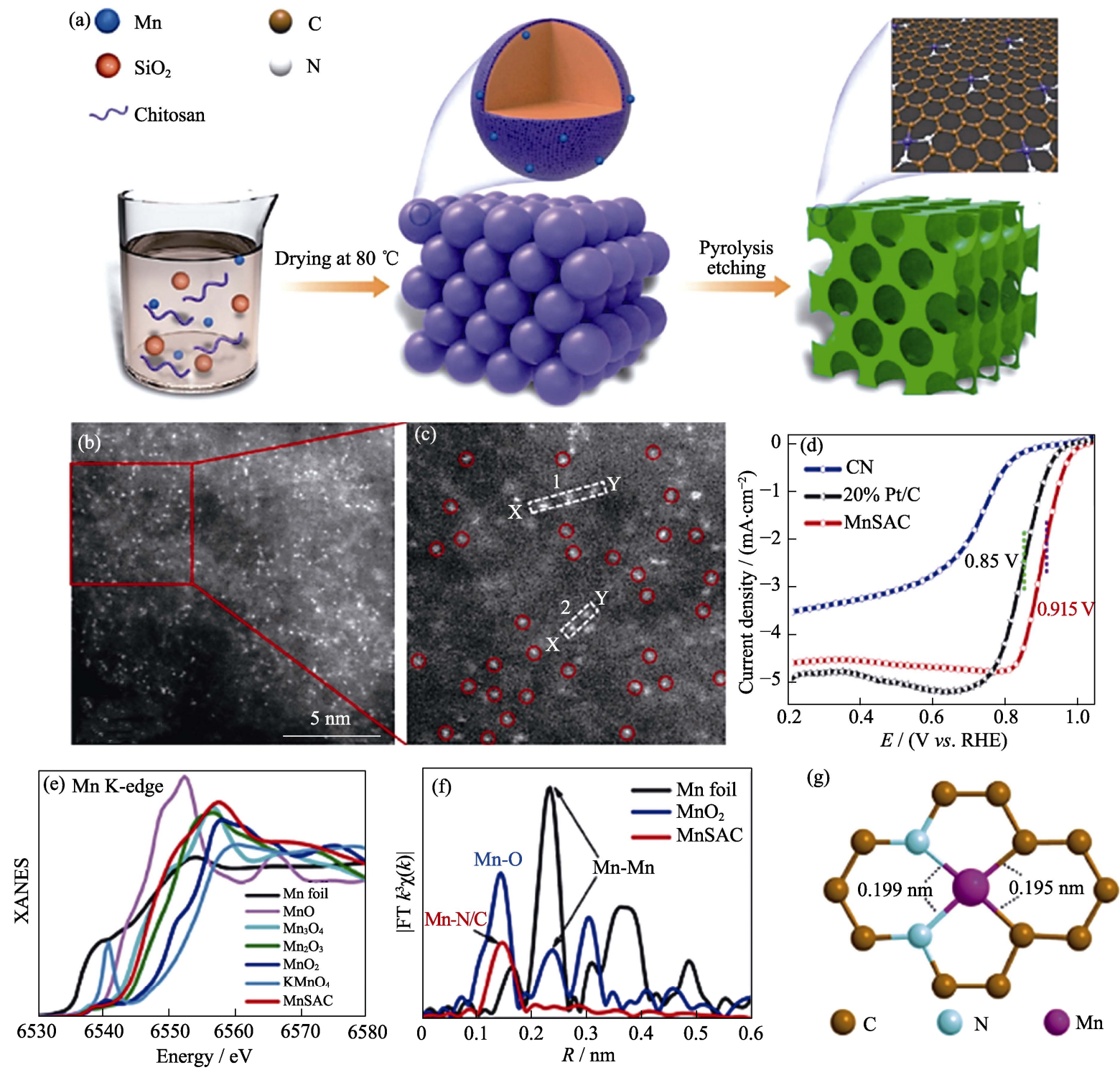

图 $10 \mathrm{Mn}-\mathrm{N}_{2} \mathrm{C}_{2}$ 的制备过程示意图以及结构表征 ${ }^{[75]}$

Fig. 10 Formation process and structural characterizations of $\mathrm{Mn}-\mathrm{N}_{2} \mathrm{C}_{2}{ }^{[75]}$

(a) Illustration of the formation of MnSAC; (b, c) HAADF-STEM images of MnSAC; (d) iR-corrected ORR polarization curves for MnSAC, CN, and 20\% Pt/C; (e) Mn K-edge XANES and (f) FT EXAFS spectra of MnSAC and their references; (g) Atomic structure model for MnSAC Colorful figures are available on website

$\mathrm{Ni}$ 等 ${ }^{[79]}$ 研究了 $\mathrm{O}$ 掺杂对 ORR 的促进作用, 首 先在 $\mathrm{Ar}$ 氛围和 $\mathrm{H}_{2}-\mathrm{Ar}$ 氛围中分别制备了 SAFe-NDC 和 SAFe-NDC-H 两种模型 Fe SACs, 在碱性环境 $\left(0.1 \mathrm{~mol} \cdot \mathrm{L}^{-1} \mathrm{KOH}\right)$ 中, 二者的半波电位均优于商业 $\mathrm{Pt} / \mathrm{C}$ 催化剂。并且, SAFe-NDC-H 的半波电位 $(0.86 \mathrm{~V}$ (vs. RHE))比 SAFe-NDC 高约 $25 \mathrm{mV}$ 。二者的表面性 质和多孔结构一致, 因此电化学活性主要受活性位 点的内在活性控制。排除 $\mathrm{N}$ 和 $\mathrm{S}$ 原子掺杂的潜在影 响后, SAFe-NDC-H 的活性较佳与其较多的 O 掺杂 有关, 碳平面上的 $\mathrm{C}=\mathrm{O}$ 可以提高单个 $\mathrm{Fe}$ 位点的催 化活性。DFT 计算结果显示, 在 $\mathrm{Fe}-\mathrm{N}_{4}$ 位点附近引 入 $\mathrm{C}=\mathrm{O}$ 后, ${ }^{*} \mathrm{OOH}$ 的形成能垒和 ${ }^{*} \mathrm{OH}$ 的还原能垒降 低, Fe- $\mathrm{N}_{4}-\mathrm{C}-\mathrm{CO}$ 的反应过电位为 $0.79 \mathrm{~V}$, 比 Fe- $\mathrm{N}_{4}$ 位
点低约 $110 \mathrm{mV}$, 表示含 $\mathrm{Fe}-\mathrm{N}_{4}-\mathrm{C}-\mathrm{CO}$ 活性位点的催 化剂更有利于氧的还原, 这与实验结果一致。

\section{4 总结与展望}

在未来清洁能源转换技术中, SACs 可能作为贵 金属催化剂的经济替代品而实现大规模制备及工业 化应用, 对于减轻催化剂对贵金属的依赖并合理利用 金属资源，从而实现高效绿色催化具有重要意义。

在催化反应方面, SACs 的应用十分广泛。根据 研究报道, 除了 ORR, SACs 对析氧反应(OER $)^{[80-82] 、}$ 析氢反应 $(\mathrm{HER})^{[83-85] 、 \mathrm{CO}_{2} \text { 还原 }}{ }^{[86-90]}$ 、电还原合成

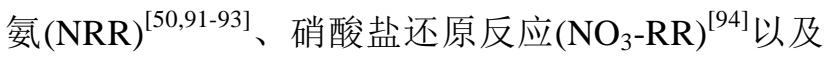


其它有机反应 ${ }^{[95-96]}$ 等均显示出优秀的催化活性。

在电化学方面, SACs 可用作电极催化剂, 提高 电化学储能装置的应用性能。以 SACs 作为正极的 质子交换膜燃料电池显示出优秀的功率密度 ${ }^{[97-98]}$ 。 引入 SACs 的金属-空气电池展示出高开路电压以及 优秀的稳定性 ${ }^{[99-105]}$ 。另外, SACs 还能够应用于锂 硫电池 ${ }^{[106-107]} 、 \mathrm{CO}_{2}$ 电池 ${ }^{[90]}$ 以及染料敏化太阳能电 池 ${ }^{[108-109]}$ 等装置中。

本文主要综述了 C-SACs 的最新研究进展。首 先介绍了 SACs 催化剂的发展和应用, 以及 C-SACs 在 ORR 中的研究进展。然后阐述了常用金属原子 (Fe、Co、 Ni) 的不足, 并介绍了其它金属单原子研 究工作。针对金属单原子的配位结构和杂原子掺杂 两个方面的研究工作展开综述。C-SACs 由于组成 和结构可控、导电性和化学稳定性优秀, 能够表现 出与商业 $\mathrm{Pt} / \mathrm{C}$ 催化剂相当的 ORR 活性。C-SACs 在 ORR 催化方面取得了许多研究成果, 这一领域 充满了机遇与挑战。

首先, 在 SACs 中金属原子的负载量一般较低, 导致产生的电流密度低, 不适合实际应用。因此, 迫 切需要发展和优化合成策略, 以获得高分散、高密 度的金属 SACs。其次, 大多数 SACs 在碱性环境下 的 ORR 性能都超过了商用 $\mathrm{Pt} / \mathrm{C}$ 催化剂, 而在酸性 环境下的 ORR 性能还有差距, 这意味着不同环境 下 SACs 的 ORR 活性差异与结构的关系需要更深 入的研究。阐明活性与结构关系大多根据理论计算 完成, 但在反应过程中, 活性中心与中间体或溶剂 分子产生吸-脱附效应时, 其结构会发生动态变化, 因此需要将原位表征技术应用到实验探究中。另外, 除了制备高活性、高稳定性的 SACs 之外, 开发低 成本和大规模合成技术也十分必要, 这直接关系到 SACs 的实际应用价值。目前报道的绝大多数 SACs 的合成策略都是复杂且耗时的。因此, 开发简单而 低廉的方法大规模制备 SACs 是实现其在工业上应 用的关键。综上所述, C-SACs 在 ORR 中显示出巨 大的潜力。随着制备技术以及表征技术的不断改进, 该催化剂具有广阔的应用以及市场前景。

\section{参考文献:}

[1] DEBE M K. Electrocatalyst approaches and challenges for automotive fuel cells. Nature, 2012, 486(7401): 43-51.

[2] BANHAM D, YE S. Current status and future development of catalyst materials and catalyst layers for proton exchange membrane fuel cells: an industrial perspective. ACS Energy Letters, 2017, 2(3): 629-638.

[3] LEE J S, TAI KIM S, CAO R, et al. Metal-air batteries with high energy density: Li-air versus Zn-air. Advanced Energy Materials, 2011, 1(1): 34-50.
[4] GUO S, ZHANG S, SUN S. Tuning nanoparticle catalysis for the oxygen reduction reaction. Angew. Chem. Int. Ed., 2013, 52(33): 8526-8544.

[5] GONG M, LI Y, WANG H, et al. An advanced Ni-Fe layered double hydroxide electrocatalyst for water oxidation. J. Am. Chem. Soc., 2013, 135(23): 8452-8455.

[6] QIAO J, LIU Y, HONG F, et al. A review of catalysts for the electroreduction of carbon dioxide to produce low-carbon fuels. Chem. Soc. Rev., 2014, 43(2): 631-675.

[7] SHAO M, CHANG Q, DODELET J P, et al. Recent advances in electrocatalysts for oxygen reduction reaction. Chem. Rev., 2016, 116(6): 3594-3657.

[8] HU B C, WU Z Y, CHU S Q, et al. $\mathrm{SiO}_{2}$-protected shell mediated templating synthesis of $\mathrm{Fe}-\mathrm{N}$-doped carbon nanofibers and their enhanced oxygen reduction reaction performance. Energy \& Environmental Science, 2018, 11(8): 2208-2215.

[9] SHARIFI T, GRACIA ESPINO E, CHEN A, et al. Oxygen reduction reactions on single- or few-atom discrete active sites for heterogeneous catalysis. Advanced Energy Materials, 2019, 10(11): 1902084.

[10] ZHU J, MU S. Defect engineering in carbon-based electrocatalysts: insight into intrinsic carbon defects. Advanced Functional Materials, 2020, 30(25): 2001097.

[11] YAN X, JIA Y, YAO X. Defects on carbons for electrocatalytic oxygen reduction. Chem. Soc. Rev., 2018, 47(20): 7628-7658.

[12] ZHAO C X, LI B Q, LIU J N, et al. Intrinsic electrocatalytic activity regulation of $\mathrm{M}-\mathrm{N}-\mathrm{C}$ single-atom catalysts for oxygen reduction reaction. Angew. Chem. Int. Ed., 2021, 60(9): 4448-4468.

[13] QIAO B, WANG A, YANG X, et al. Single-atom catalysis of CO oxidation using $\mathrm{Pt}_{1} / \mathrm{FeO}_{x}$. Nat. Chem., 2011, 3(8): 634-641.

[14] DENG J, LI H, XIAO J, et al. Triggering the electrocatalytic hydrogen evolution activity of the inert two-dimensional $\mathrm{MoS}_{2}$ surface via single-atom metal doping. Energy \& Environmental Science, 2015, 8(5): 1594-1601.

[15] LANG R, LI T, MATSUMURA D, et al. Hydroformylation of olefins by a rhodium single-atom catalyst with activity comparable to $\mathrm{RhCl}\left(\mathrm{PPh}_{3}\right)_{3}$. Angew. Chem. Int. Ed., 2016, 55(52): 16054-16058.

[16] LIN J, WANG A, QIAO B, et al. Remarkable performance of $\mathrm{Ir}_{1} / \mathrm{FeO}_{x}$ single-atom catalyst in water gas shift reaction. J. Am. Chem. Soc., 2013, 135(41): 15314-15317.

[17] LIU P, ZHAO Y, QIN R, et al. Photochemical route for synthesizing atomically dispersed palladium catalysts. Science, 2016, 352(6287): 797-801.

[18] CHEN Y, JI S, WANG Y, et al. Isolated single iron atoms anchored on $\mathrm{N}$-doped porous carbon as an efficient electrocatalyst for the oxygen reduction reaction. Angew. Chem. Int. Ed., 2017, 56(24): 6937-6941.

[19] ZHANG C, SHA J, FEI H, et al. Single-atomic ruthenium catalytic sites on nitrogen-doped graphene for oxygen reduction reaction in acidic medium. ACS Nano, 2017, 11(7): 6930-6941.

[20] CHEN K, LIU K, AN P, et al. Iron phthalocyanine with coordination induced electronic localization to boost oxygen reduction reaction. Nat. Commun., 2020, 11(1): 4173.

[21] ZHU J, MU S. Defect engineering in the carbon-based electrocatalysts: insight into the intrinsic carbon defects. Advanced Functional Materials, 2020, 30(25): 2001097.

[22] BAI X, SHI Y, GUO J, et al. Catalytic activities enhanced by abundant structural defects and balanced $\mathrm{N}$ distribution of $\mathrm{N}$-doped graphene in oxygen reduction reaction. Journal of Power Sources, 2016, 306: 85-91.

[23] LI Y, WEN H, YANG J, et al. Boosting oxygen reduction catalysis with $\mathrm{N}, \mathrm{F}$, and $\mathrm{S}$ tri-doped porous graphene: tertiary N-precursors regulates the constitution of catalytic active sites. Carbon, 2019, 
142: $1-12$.

[24] QIU X, YAN X, PANG $\mathrm{H}$, et al. Isolated Fe single atomic sites anchored on highly steady hollow graphene nanospheres as an efficient electrocatalyst for the oxygen reduction reaction. Adv. Sci., 2019, 6(2): 1801103.

[25] WU Y, ZHOU H, YANG T, et al. Negative pressure pyrolysis induced highly accessible single sites dispersed on 3D graphene frameworks for enhanced oxygen reduction. Angew. Chem. Int. Ed., 2020, 59(46): 20465-20469.

[26] DENG Y, CHI B, LI J, et al. Atomic Fe-doped MOF-derived carbon polyhedrons with high active-center density and ultra-high performance toward pem fuel cells. Advanced Energy Materials, 2019, 9(13): 1802856.

[27] HUANG K, ZHANG L, XU T, et al. $-60{ }^{\circ} \mathrm{C}$ solution synthesis of atomically dispersed cobalt electrocatalyst with superior performance. Nat. Commun., 2019, 10(1): 606.

[28] WALLING C. Fentons reagent revisited. Accounts of Chemical Research, 1975, 8(4): 125-131.

[29] ZHONG Y, LIANG X, HE Z, et al. The constraints of transition metal substitutions ( $\mathrm{Ti}, \mathrm{Cr}, \mathrm{Mn}, \mathrm{Co}$ and $\mathrm{Ni}$ ) in magnetite on its catalytic activity in heterogeneous Fenton and UV/Fenton reaction: from the perspective of hydroxyl radical generation. Applied Catalysis B: Environmental, 2014, 150: 612-618.

[30] QU Y, LI Z, CHEN W, et al. Direct transformation of bulk copper into copper single sites via emitting and trapping of atoms. Nature Catalysis, 2018, 1(10): 781-786.

[31] JIANG Z, SUN W, SHANG H, et al. Atomic interface effect of a single atom copper catalyst for enhanced oxygen reduction reactions. Energy \& Environmental Science, 2019, 12(12): 3508-3514.

[32] CUI L, CUI L, LI Z, et al. A copper single-atom catalyst towards efficient and durable oxygen reduction for fuel cells. Journal of Materials Chemistry A, 2019, 7(28): 16690-16695.

[33] BAI L, HOU C, WEN X, et al. Catalysis of oxygen reduction reaction on atomically dispersed copper- and nitrogen-codoped graphene. ACS Applied Energy Materials, 2019, 2(7): 4755-4762.

[34] WANG D, AO C, LIU X, et al. Coordination-engineered Cu- $\mathrm{N}_{x}$ single-site catalyst for enhancing oxygen reduction reaction. ACS Applied Energy Materials, 2019, 2(9): 6497-6504.

[35] HAN G, ZHENG Y, ZHANG X, et al. High loading single-atom $\mathrm{Cu}$ dispersed on graphene for efficient oxygen reduction reaction. Nano Energy, 2019, 66: 104088.

[36] WAGH N K, SHINDE S S, LEE C H, et al. Densely colonized isolated $\mathrm{Cu}-\mathrm{N}$ single sites for efficient bifunctional electrocatalysts and rechargeable advanced Zn-air batteries. Applied Catalysis B: Environmental, 2020, 268: 118746.

[37] YANG Z, CHEN B, CHEN W, et al. Directly transforming copper (I) oxide bulk into isolated single-atom copper sites catalyst through gas-transport approach. Nat. Commun., 2019, 10(1): 3734

[38] LI J, CHEN M, CULLEN D A, et al. Atomically dispersed manganese catalysts for oxygen reduction in proton-exchange membrane fuel cells. Nature Catalysis, 2018, 1(12): 935-945.

[39] YANG Y, MAO K, GAO S, et al. O-, N-atoms-coordinated Mn cofactors within a graphene framework as bioinspired oxygen reduction reaction electrocatalysts. Adv. Mater., 2018, 30(28): e1801732.

[40] ZHU X, AMAL R, LU X. N, P co-coordinated manganese atoms in mesoporous carbon for electrochemical oxygen reduction. Small, 2019, 15(29): e1804524.

[41] BAI L, DUAN Z, WEN X, et al. Atomically dispersed manganesebased catalysts for efficient catalysis of oxygen reduction reaction. Applied Catalysis B: Environmental, 2019, 257: 117930.

[42] LIN Z, HUANG H, CHENG L, et al. Atomically dispersed Mn within carbon frameworks as high-performance oxygen reduction electrocatalysts for zinc-air battery. ACS Sustainable Chemistry \& Engineering, 2019, 8(1): 427-434.

[43] CHEN Z, GONG W, LIU Z, et al. Coordination-controlled single-atom tungsten as a non-3d-metal oxygen reduction reaction electrocatalyst with ultrahigh mass activity. Nano Energy, 2019, 60 394-403.

[44] SONG P, LUO M, LIU X, et al. Zn single atom catalyst for highly efficient oxygen reduction reaction. Advanced Functional Materials, 2017, 27(28): 1100802.

[45] LI J, CHEN S, YANG N, et al. Ultrahigh-loading zinc single-atom catalyst for highly efficient oxygen reduction in both acidic and alkaline media. Angew. Chem. Int. Ed., 2019, 58(21): 7035-7039.

[46] LUO E, ZHANG H, WANG X, et al. Single-atom $\mathrm{Cr}^{-\mathrm{N}_{4}}$ sites designed for durable oxygen reduction catalysis in acid media. Angew. Chem. Int. Ed., 2019, 58(36): 12469-12475.

[47] CALLE-VALLEJO F, IGNACIO MARTINEZ J, ROSSMEISL J. Density functional studies of functionalized graphitic materials with late transition metals for oxygen reduction reactions. Physical Chemistry Chemical Physics, 2011, 13(34): 15639-15643.

[48] LIU S, LI Z, WANG C, et al. Turning main-group element magnesium into a highly active electrocatalyst for oxygen reduction reaction. Nat. Commun., 2020, 11(1): 938.

[49] CAO Y, GAO Y, ZHOU H, et al. Highly efficient ammonia synthesis electrocatalyst: single $\mathrm{Ru}$ atom on naturally nanoporous carbon materials. Advanced Theory and Simulations, 2018, 1(5): 1800018.

[50] YU B, LI H, WHITE J, et al. Tuning the catalytic preference of ruthenium catalysts for nitrogen reduction by atomic dispersion. Advanced Functional Materials, 2019, 30(6): 1905665.

[51] JI S, CHEN Y, FU Q, et al. Confined pyrolysis within metalorganic frameworks to form uniform $\mathrm{Ru}_{3}$ clusters for efficient oxidation of alcohols. J. Am. Chem. Soc., 2017, 139(29): 9795-9798.

[52] XIAO M, GAO L, WANG Y, et al. Engineering energy level of metal center: Ru single-atom site for efficient and durable oxygen reduction catalysis. J. Am. Chem. Soc., 2019, 141(50): 19800-19806.

[53] XIAO M, ZHU J, LI G, et al. A single-atom iridium heterogeneous catalyst in oxygen reduction reaction. Angew. Chem. Int. Ed., 2019, 58(28): 9640-9645.

[54] LIU Q, LI Y, ZHENG L, et al. Sequential synthesis and active-site coordination principle of precious metal single-atom catalysts for oxygen reduction reaction and PEM fuel cells. Advanced Energy Materials, 2020, 10(20): 2000689.

[55] LIU W, JI J, YAN X, et al. A cascade surface immobilization strategy to access high-density and closely distanced atomic $\mathrm{Pt}$ sites for enhancing alkaline hydrogen evolution reaction. Journal of Materials Chemistry A, 2020, 8(10): 5255-5262.

[56] ZHANG L, DOYLE-DAVIS K, SUN X. Pt-based electrocatalysts with high atom utilization efficiency: from nanostructures to single atoms. Energy \& Environmental Science, 2019, 12(2): 492-517.

[57] CHOI C H, KIM M, KWON H C, et al. Tuning selectivity of electrochemical reactions by atomically dispersed platinum catalyst. Nat. Commun., 2016, 7: 10922.

[58] SONG X, LI N, ZHANG H, et al. Promotion of hydrogen peroxide production on graphene-supported atomically dispersed platinum: effects of size on oxygen reduction reaction pathway. Journal of Power Sources, 2019, 435: 226771.

[59] LIU J, JIAO M, MEI B, et al. Carbon-supported divacancyanchored platinum single-atom electrocatalysts with superhigh Pt utilization for the oxygen reduction reaction. Angew. Chem. Int. Ed., 2019, 58(4): 1163-1167.

[60] LIU J, JIAO M, LU L, et al. High performance platinum single atom electrocatalyst for oxygen reduction reaction. Nat. Commun., 
2017, 8: 15938.

[61] ZHANG L, LIU H, LIU S, et al. Pt/Pd single-atom alloys as highly active electrochemical catalysts and the origin of enhanced activity. ACS Catalysis, 2019, 9(10): 9350-9358.

[62] ZHANG Q, QIN X X, DUAN-MU F P, et al. Isolated platinum atoms stabilized by amorphous tungstenic acid: metal-support interaction for synergistic oxygen activation. Angew. Chem. Int. Ed., 2018, 57(30): 9351-9356.

[63] ZHU Y, SOKOLOWSKI J, SONG X, et al. Engineering local coordination environments of atomically dispersed and heteroatomcoordinated single metal site electrocatalysts for clean energyconversion. Advanced Energy Materials, 2020, 10(11): 1902844.

[64] HAN X, LING X, YU D, et al. Atomically dispersed binary Co-Ni sites in nitrogen-doped hollow carbon nanocubes for reversible oxygen reduction and evolution. Adv. Mater., 2019, 31(49): e1905622.

[65] YUAN K, LUTZENKIRCHEN-HECHT D, LI L, et al. Boosting oxygen reduction of single iron active sites via geometric and electronic engineering: nitrogen and phosphorus dual coordination. J. Am. Chem. Soc., 2020, 142(5): 2404-2412.

[66] ZHAO Y M, ZHANG P C, XU C, et al. Design and preparation of Fe- $\mathrm{N}_{5}$ catalytic sites in single-atom catalysts for enhancing the oxygen reduction reaction in fuel cells. ACS Appl. Mater. Interfaces, 2020, 12(15): 17334-17342.

[67] LIN Y, LIU P, VELASCO E, et al. Fabricating single-atom catalysts from chelating metal in open frameworks. Adv. Mater., 2019, 31(18): e1808193.

[68] SUN T, ZHANG P, CHEN W, et al. Single iron atoms coordinated to $g-\mathrm{C}_{3} \mathrm{~N}_{4}$ on hierarchical porous $\mathrm{N}$-doped carbon polyhedra as a high-performance electrocatalyst for the oxygen reduction reaction. Chem. Commun., 2020, 56(5): 798-801.

[69] ZHANG J, ZHANG M, ZENG Y, et al. Single Fe atom on hierarchically porous $\mathrm{S}$, N-codoped nanocarbon derived from porphyra enable boosted oxygen catalysis for rechargeable Zn-air batteries. Small, 2019, 15(24): e1900307.

[70] ZHANG J, ZHAO Y, CHEN C, et al. Tuning the coordination environment in single-atom catalysts to achieve highly efficient oxygen reduction reactions. J. Am. Chem. Soc., 2019, 141(51): 20118-20126.

[71] WEI X, ZHENG D, ZHAO M, et al. Cross-linked polyphosphazene hollow nanosphere-derived N/P-doped porous carbon with single nonprecious metal atoms for the oxygen reduction reaction. Angew. Chem. Int. Ed., 2020, 59(34): 14639-14646.

[72] CHEN P, ZHANG N, ZHOU T, et al. Tailoring electronic structure of atomically dispersed metal- $\mathrm{N}_{3} \mathrm{~S}_{1}$ active sites for highly efficient oxygen reduction catalysis. ACS Materials Letters, 2019, 1(1): 139-146.

[73] MA S, HAN Z, LENG K, et al. Ionic exchange of metal-organic frameworks for constructing unsaturated copper single-atom catalysts for boosting oxygen reduction reaction. Small, 2020, 16(23): e2001384.

[74] SUN T, LI Y, CUI T, et al. Engineering of coordination environment and multiscale structure in single-site copper catalyst for superior electrocatalytic oxygen reduction. Nano Lett., 2020, 20(8): 6206-6214.

[75] SHANG H, SUN W, SUI R, et al. Engineering isolated $\mathrm{Mn}-\mathrm{N}_{2} \mathrm{C}_{2}$ atomic interface sites for efficient bifunctional oxygen reduction and evolution reaction. Nano Lett., 2020, 20(7): 5443-5450.

[76] SUN H, WANG M, DU X, et al. Modulating the d-band center of boron doped single-atom sites to boost the oxygen reduction reaction. Journal of Materials Chemistry A, 2019, 7(36): 20952-20957.

[77] SUN H, LIU S, WANG M, et al. Updating the intrinsic activity of a single-atom site with a $\mathrm{P}-\mathrm{O}$ bond for a rechargeable Zn-air battery. ACS Appl. Mater. Interfaces, 2019, 11(36): 33054-33061.
[78] CHEN Y, JI S, ZHAO S, et al. Enhanced oxygen reduction with single-atomic-site iron catalysts for a zinc-air battery and hydrogen-air fuel cell. Nat. Commun., 2018, 9(1): 5422.

[79] NI W, GAO Y, ZHANG Y, et al. O-doping boosts the electrochemical oxygen reduction activity of a single Fe site in hydrophilic carbon with deep mesopores. ACS Appl. Mater. Interfaces, 2019, 11(49): 45825-45831.

[80] CAO L, LUO Q, CHEN J, et al. Dynamic oxygen adsorption on single-atomic ruthenium catalyst with high performance for acidic oxygen evolution reaction. Nat. Commun., 2019, 10(1): 4849.

[81] XU Y, ZHANG W, LI Y, et al. A general bimetal-ion adsorption strategy to prepare nickel single atom catalysts anchored on graphene for efficient oxygen evolution reaction. Journal of Energy Chemistry, 2020, 43: 52-57.

[82] YAO Y, HU S, CHEN W, et al. Engineering the electronic structure of single atom Ru sites via compressive strain boosts acidic water oxidation electrocatalysis. Nature Catalysis, 2019, 2(4): 304-313.

[83] SHI Y, HUANG W M, LI J, et al. Site-specific electrodeposition enables self-terminating growth of atomically dispersed metal catalysts. Nat. Commun., 2020, 11(1): 4558.

[84] HOSSAIN M D, LIU Z, ZHUANG M, et al. Rational design of graphene-supported single atom catalysts for hydrogen evolution reaction. Advanced Energy Materials, 2019, 9(10): 1803689.

[85] YUAN S, PU Z, ZHOU H, et al. A universal synthesis strategy for single atom dispersed cobalt/metal clusters heterostructure boosting hydrogen evolution catalysis at all pH values. Nano Energy, 2019, 59: 472-480.

[86] WANG G, HE C T, HUANG R, et al. Photoinduction of Cu single atoms decorated on UiO-66- $\mathrm{NH}_{2}$ for enhanced photocatalytic reduction of $\mathrm{CO}_{2}$ to liquid fuels. J. Am. Chem. Soc., 2020, 142(45): 19339-19345.

[87] LI Y, WANG S, WANG X S, et al. Facile top-down strategy for direct metal atomization and coordination achieving a high turnover number in $\mathrm{CO}_{2}$ photoreduction. J. Am. Chem. Soc., 2020, 142(45): 19259-19267.

[88] FAN Q, HOU P, CHOI C, et al. Activation of Ni particles into single Ni-N atoms for efficient electrochemical reduction of $\mathrm{CO}_{2}$. Advanced Energy Materials, 2019, 10(5): 1903068.

[89] LI Y, HAO J, SONG $\mathrm{H}$, et al. Selective light absorber-assisted single nickel atom catalysts for ambient sunlight-driven $\mathrm{CO}_{2}$ methanation. Nat. Commun., 2019, 10(1): 2359.

[90] ZHENG W, YANG J, CHEN $\mathrm{H}$, et al. Atomically defined undercoordinated active sites for highly efficient $\mathrm{CO}_{2}$ electroreduction. Advanced Functional Materials, 2020, 30(4): 1907658.

[91] TAO H, CHOI C, DING L X, et al. Nitrogen fixation by $\mathrm{Ru}$ single-atom electrocatalytic reduction. Chem., 2019, 5(1): 204-214.

[92] ZANG W, YANG T, ZOU H, et al. Copper single atoms anchored in porous nitrogen-doped carbon as efficient $\mathrm{pH}$-universal catalysts for the nitrogen reduction reaction. ACS Catalysis, 2019, 9(11): 10166-10173.

[93] HUANG Y, YANG T, YANG L, et al. Graphene-boron nitride hybrid-supported single Mo atom electrocatalysts for efficient nitrogen reduction reaction. Journal of Materials Chemistry A, 2019, 7(25): 15173-15180.

[94] ZHU T, CHEN Q, LIAO P, et al. Single-atom Cu catalysts for enhanced electrocatalytic nitrate reduction with significant alleviation of nitrite production. Small, 2020, 16: 2004526.

[95] GAN G, LI X, WANG L, et al. Active sites in single-atom Fe- $\mathrm{N}_{x}-\mathrm{C}$ nanosheets for selective electrochemical dechlorination of 1,2-dichloroethane to ethylene. ACS Nano, 2020, 14(8): 9929-9937.

[96] XIONG Y, SUN W, XIN P, et al. Gram-scale synthesis of high-loading single-atomic-site Fe catalysts for effective epoxidation of 
styrene. Adv. Mater., 2020, 32(34): e2000896.

[97] WAN X, LIU X, LI Y, et al. Fe-N-C electrocatalyst with dense active sites and efficient mass transport for high-performance proton exchange membrane fuel cells. Nature Catalysis, 2019, 2(3): 259-268.

[98] CHENG Y, HE S, LU S, et al. Iron single atoms on graphene as nonprecious metal catalysts for high-temperature polymer electrolyte membrane fuel cells. Advanced Science, 2019, 6(10): 1802066.

[99] LI B, ZHAO C, CHEN S, et al. Framework-porphyrin-derived single-atom bifunctional oxygen electrocatalysts and their applications in Zn-air batteries. Adv. Mater., 2019, 31(19): 1900592.

[100] HAN J, MENG X, LU L, et al. Single-atom Fe- $\mathrm{N}_{x}-\mathrm{C}$ as an efficient electrocatalyst for zinc-air batteries. Advanced Functional Materials, 2019, 29(41): 1808872.

[101] CHEN L, ZHANG Y, DONG L, et al. Synergistic effect between atomically dispersed $\mathrm{Fe}$ and Co metal sites for enhanced oxygen reduction reaction. Journal of Materials Chemistry A, 2020, 8(8): 4369-4375.

[102] XIAO M, XING Z, JIN Z, et al. Preferentially engineering FeN $\mathrm{N}_{4}$ edge sites onto graphitic nanosheets for highly active and durable oxygen electrocatalysis in rechargeable Zn-air batteries. Adv. Mater., 2020, 32(49): 2004900.

[103] ZHANG Z, ZHAO X, XI S, et al. Atomically dispersed cobalt trifunctional electrocatalysts with tailored coordination environment for flexible rechargeable Zn-air battery and self-driven water splitting. Advanced Energy Materials, 2020, 10(48): 2002896.

[104] ZHOU Y, TAO X, CHEN G, et al. Multilayer stabilization for fabricating high-loading single-atom catalysts. Nat. Commun., 2020, 11(1): 5892.

[105] NOH W Y, KIM E M, KIM K Y, et al. Immobilizing single atom catalytic sites onto highly reduced carbon hosts: $\mathrm{Fe}-\mathrm{N}_{4} / \mathrm{CNT}$ as a durable oxygen reduction catalyst for Na-air batteries. Journal of Materials Chemistry A, 2020, 8(36): 18891-18902.

[106] ZHANG L, LIU D, MUHAMMAD Z, et al. Single nickel atoms on nitrogen-doped graphene enabling enhanced kinetics of lithium-sulfur batteries. Adv. Mater., 2019, 31(40): 1903955.

[107] LI B Q, KONG L, ZHAO C X, et al. Expediting redox kinetics of sulfur species by atomic-scale electrocatalysts in lithium-sulfur batteries. InforMat, 2019, 1(4): 533-541.

[108] YANG W, XU X, HOU L, et al. Nitrogen-enriched hollow carbon spheres coupled with efficient $\mathrm{Co}-\mathrm{N}_{x}-\mathrm{C}$ species as cathode catalysts for triiodide reduction in dye-sensitized solar cells. ACS Sustainable Chemistry \& Engineering, 2019, 7(2): 2679-2685.

[109] YANG W, LI Z, XU X, et al. Atomic N-coordinated cobalt sites within nanomesh graphene as highly efficient electrocatalysts for triiodide reduction in dye-sensitized solar cells. Chemical Engineering Journal, 2018, 349: 782-790. 